\title{
2 Comb-like dextran copolymers: A versatile strategy to 3 coat highly porous MOF nanoparticles with a PEG 4 shell
}

5 Giovanna Cutrone ${ }^{1}+$, Jingwen Qiu ${ }^{2}+$, Mario Menendez-Miranda ${ }^{2}$, Juan M. Casas-Solvas ${ }^{1}$, Ahmet

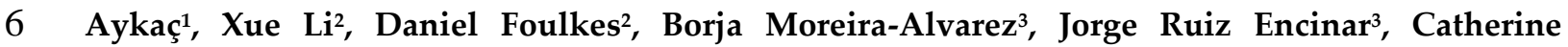
7 Ladavière ${ }^{4}$, Didier Desmaële ${ }^{5}$, Antonio Vargas-Berenguel ${ }^{1, *}$ and Ruxandra Gref ${ }^{2, *}$

1 Department of Chemistry and Physics, University of Almería, Ctra. de Sacramento s/n, 04120 Almería, Spain; cutrone@ual.es (G.C.); jmcasas@ual.es (J.M.C.-S.); ahmet.aykac@ikc.edu.tr (A.A.)

2 Institute of Molecular Sciences, UMR CNRS 8214, Université Paris-Sud, Université Paris Saclay, 91400 Orsay, France; jingwen.qiu@u-psud.fr (J.Q.); mario.menendez-miranda@u-psud.fr (M.M.-M.); xue.li@u-psud.fr (X.L.); Daniel.Foulkes@liverpool.ac.uk (D.F.)

3 Department of Physical and Analytical Chemistry, University of Oviedo, Julián Clavería 8, 33006 Oviedo, Spain; borjamoreira@gmail.com (B.M.-A.); ruizjorge@uniovi.es (J.R.E.)

4 University of Lyon, CNRS, UMR 5223, IMP, 15 bd André Latarjet, F-69622 Villeurbanne, France; catherine.ladaviere@univ-lyon1.fr

5 Institut Galien Paris-Sud, UMR 8612, CNRS, Université Paris-Sud, Faculté de Pharmacie, 5 rue JB Clément, 92296 Châtenay-Malabry, France; didier.desmaele@u-psud.fr

* Correspondence: ruxandra.gref@u-psud.fr (R.G.); Tel.: +33 169158234 (R.G.)

(A. V-B.); Tel..+34 950015315 (A. V-B.);

+ These authors equally contributed to this work.

Received: date; Accepted: date; Published: date

Abstract:

Nanoparticles made of metal-organic frameworks (nanoMOFs) are becoming of increasing interest as drug carriers. However, preventing nanoMOFs recognition and clearance by the innate immune system, a prerequisite for biomedical applications, presents an important challenge. In this study we provide a proof of concept that the outer surface of biocompatible iron-based nanoMOFs can be functionalized in a rapid, organic solvent-free and non-covalent manner using a novel family of comb-like copolymers made of dextran (DEX) grafted with both poly(ethylene glycol) (PEG) and alendronate (ALN) moieties. We describe the synthesis and full characterization of DEX-PEG-ALN copolymers by click chemistry, with control of both the amount of grafted PEG and ALN moieties. The copolymers, freely soluble in aqueous media, were used to directly coat the nanoMOFs in water by simple incubation at room temperature. The coating procedure did not affect the nanoMOFs' morphology nor their crystalline structure. As strong iron complexing groups, the ALN moieties ensured multiple cooperative anchoring of the copolymers to the nanoMOFs surface, resulting in stable coatings that substantially decreased their internalization by macrophages in vitro, providing new perspectives for biomedical applications. 
Keywords: metal organic frameworks; nanoparticles; surface modification; dextran; poly(ethylene glycol); macrophage uptake; click chemistry.

\section{Introduction}

Metal-organic frameworks (MOFs) are one of the latest classes of ordered porous solids which have attracted growing interest since their discovery in 1989 [1], in reason of their remarkable versatility. Indeed, almost any metal could be associated to polycomplexing linkers such as carboxylates, phosphonates, sulfonates or imidazolates leading to the discovery of thousands of MOFs with a variety of pore sizes and shapes [2,3]. Among the MOF family, nanosized MOFs (nanoMOFs) based on porous iron(III) polycarboxylates have emerged as an important class of biodegradable and non-toxic $[4,5]$ materials that can be loaded with exceptional quantities (within the $20-70 \mathrm{wt} \%$ range) of a large variety of therapeutic agents $[4,6]$. This paved the way to novel perspectives in terms of targeted delivery of drugs [7,8] and theranostics [4]. For biomedical applications, it is of utmost importance to engineer the surface of the nanoMOFs, since the in vivo fate of any nanoparticle in the living body (biodistribution, pharmacokinetics and targeting abilities) depends upon its surface physicochemical properties. For instance, it was shown that surface functionalization with hydrophilic polymers such as poly(ethylene glycol) (PEG) in a "brush" configuration could dramatically extend the blood circulation times of nanoparticles by mitigating their recognition by the reticuloendothelial system $[9,10]$. However, to date, Doxil ${ }^{\circledR}$ represents the only FDA-approved PEGylated liposome-based nanocarrier of anticancer drug (Doxorubicin) [11].

Functionalization of nanoMOF surfaces will be critical to their success as potential nanocarriers in therapeutic contexts. However, thus far, only a limited number of cases have been reported that have aimed to modify the surface of nanoMOFs with PEG shells [12-16]. As compared to dense nanoparticles made of biodegradable polymers or liposomes, the porous surface of MOFs is a challenging surface to functionalize. Indeed, it was reported that PEG chains are able to penetrate within the pores, blocking them and/or decreasing the drug loading capacity [12]. To avoid PEG penetration into the highly porous MOFs, PEG-based shells were formed by GraftFast, a method involving polymerization of acryl PEGs [14]. By this way, PEG derivatives with initial molecular weights of 480, 2000 and 5000 Da were polymerized leading to stable coatings. However, the molecular weight of the resulting PEG-based copolymer could not be efficiently controlled. Another recent strategy employed cyclodextrin (CD) derivatives, which were first adsorbed onto the MOFs' surfaces prior to complex formation with PEG grafted with adamantane moieties [14]. However, the two-step procedure required by this method resulted in a difficulty to control the quantity of grafted PEG.

Moreover, no study has yet demonstrated that a PEG coating on nanoMOFs could effectively reduce their reticuloendothelial sequestration. In this context, there is still a clear demand to engineer versatile PEG-based coatings onto the nanoMOF surface, with proof of concept, by reducing uptake by macrophages. Here we address this challenge by using a novel family of copolymers, synthesized by grafting onto a dextran (DEX) backbone two types of moieties: i) PEG chains to avoid macrophage uptake and ii) alendronate (ALN) to spontaneously coordinate to the nanoMOFs surface. Iron trimesate MIL-100(Fe) (MIL stands for Material of the Institute Lavoisier) nanoMOFs were selected as core materials, in reason of their biodegradability, capacity to incorporate a series of drugs (antibiotics [17], anticancer drugs [18,19], anti-infective agents [6,20]) reaching unprecedented payloads together with controlled releases, versatility in terms of drug loadings and lack of in vivo toxicity $[5,21]$.

Here we show the possibility to achieve stable coatings by a straightforward method, based on cooperative interactions between the ALN moieties and the external surface of MIL-100(Fe) nanoMOFs. Thus, we describe the synthetic strategies to control both PEG and ALN densities on the 
DEX backbone, as well as the convenient one-step method to coat the nanoMOFs. The PEG "brush" efficiently reduced macrophage uptake as demonstrated by both microscopic investigations and quantitative ICP-MS determination of the amount of internalized nanoMOFs.

\section{Materials and Methods}

\subsection{Chemicals and general methods}

Thin layer chromatography (TLC) was performed on Merck silica gel $60 \mathrm{~F}_{254}$ aluminum sheets and developed by UV-vis light, iodine, $5 \% \mathrm{v} / \mathrm{v}$ sulfuric acid in ethanol, $5 \% \mathrm{w} / \mathrm{v}$ phosphomolybdic acid in ethanol, and $1 \% \mathrm{w} / \mathrm{v}$ potassium permanganate in aqueous $0.1 \% \mathrm{w} / \mathrm{v} \mathrm{NaOH}$ containing $7 \% \mathrm{w} / \mathrm{v}$ potassium carbonate, depending on the case. Flash column chromatography was performed on Merck silica gel (230-400 mesh, ASTM). Infrared spectra were recorded on a Bruker Alpha FTIR equipped with a Bruker universal ATR sampling accessory. ${ }^{1} \mathrm{H},{ }^{13} \mathrm{C},{ }^{31} \mathrm{P}$ and $2 \mathrm{D}$ NMR spectra were recorded on a Bruker Avance III HD $600 \mathrm{MHz}$ spectrometer equipped with a QCI ${ }^{1} \mathrm{H} /{ }^{13} \mathrm{C} /{ }^{15} \mathrm{~N} /{ }^{13} \mathrm{P}$ proton-optimized quadrupole inverse cryoprobe with ${ }^{1} \mathrm{H}$ and ${ }^{13} \mathrm{C}$ cryochannels, or a Bruker Nanobay Avance III HD $300 \mathrm{MHz}$ spectrometer equipped with a QNP ${ }^{1} \mathrm{H} /{ }^{13} \mathrm{C} /{ }^{19} \mathrm{~F} /{ }^{13} \mathrm{P}$ probe, depending on the sample. Standard Bruker software was used for acquisition and processing routines. Chemical shifts $(\delta)$ are given in parts per million (ppm) and referenced to internal tetramethylsilane (TMS) signal $\left(\delta_{\mathrm{H}}\right.$, $\delta c 0.00)$. $J$ values are given in hertz (Hz). ESI-TOF mass spectra were recorded on a Agilent LC/MSDTOF spectrometer in both positive and negative modes. Syringe filtering was conducted using nylon $0.45 \mu \mathrm{m}$ Milipore Millex ${ }^{\circledR}$ syringe-driven filter units. Dialysis was performed using Medicell Membranes Ltd 12000-14000 Da molecular weight cutoff (MWCO) Visking dialysis tubing. Elemental analyses were recorded on an Elementar Vario Micro CHNS analyzer. HR-ICP-MS results were obtained by using a Termo Finningan magnetic sector field ELEMENT 2 inductively coupled plasma mass spectrometer. A Hanna HI $98192 \mathrm{EC} / \mathrm{TDS} / \mathrm{NaCl} /$ Resistivity meter was employed to monitor dialysate solutions conductivity during dialysis.

6-Bromohexanoic acid (Aldrich, 97\%), sodium azide (Panreac, 99\%), N-hydroxysuccinimide (NHS, Aldrich, 98\%), $N$-(3-dimethylaminopropyl)-N'-ethylcarbodiimide hydrochloride (EDC, Fluka, $\geq 98 \%$ ), alendronic acid monosodium salt trihydrate (CarboSynth, purum), poly(ethylene glycol) methyl ether (MeOPEG45OH, Aldrich, Mn 2000), 4-dimethylaminopyridine (DMAP, Fluka, $\geq 98 \%$ ), methanesulfonyl chloride ( $\mathrm{MsCl}$, Fluka, 299\%), 1,1'-carbonyldiimidazole (CDI, Acros, 97\%), propargylamine (Aldrich, 98\%), anhydrous copper(II) sulfate (Fluka, 98\%), (+)-sodium L-ascorbate (Sigma, BioXtra, $\geq 99 \%$ ), and ethylenediaminetetraacetic acid disodium salt dihydrate (EDTA, Fluka, purum) were purchased from commercial sources and used without further purification otherwise indicated. Anhydrous LiCl (Sigma-Aldrich, 99\%) and dextran T-40 (Pharmacosmos, purum, Nominative Mw $40000 \mathrm{Da}$ ) were purchased from commercial sources and dried at $80{ }^{\circ} \mathrm{C}$ under high vacuum for $48 \mathrm{~h}$ in the presence of $\mathrm{P}_{2} \mathrm{O}_{5}$ prior to use. Triethylamine (Sigma-Aldrich, $>99 \%$ ) and organic solvents were dried according to literature procedures [22]. Dry DMF (AcroSeal, 99.8\%, over molecular sieves) was purchased from Acros. Iron (III) chloride hexahydrate (Alfa Aesar, Schiltigheim, France, 98\%), 1,3,5-benzenetricarboxylic acid (BTC, Sigma-Aldrich, Saint-QuentinFallavier, France, 95\%) and absolute ethanol (Carlo Erba, Val-de-Reuil, France, 99\%,) were used for the synthesis of nanoMOFs. Potassium chloride (Sigma-Aldrich, XX\%) was used for nanoMOFs Zeta potential (ZP) measurements. Human serum albumin (HSA, Sigma-Aldrich) and bicinchoninic acid (BCA) protein assay kit (Pierce ${ }^{\mathrm{TM}}$ Thermo Fisher) was used for HSA adsorption test. Deionized Milli$\mathrm{Q}$ water was obtained from a Millipore apparatus with a $0.22 \mu \mathrm{m}$ filter.

Number average molecular weight analysis. The number average molecular weight $(M n)$ and the molar-mass dispersity values of dextran derivatives $\mathbf{5}$ and $\mathbf{7}$ were measured by using a SEC column (TSKgel G2500PW and G6000PW columns) coupled with a differential refractometer (RI, Optilab T-rEX, Wyatt Technology) with a laser at $\lambda=658 \mathrm{~nm}$, thermostated at $25^{\circ} \mathrm{C}$, and a multi-angle 
laser light scattering instrument (MALLS, HELEOS II, Wyatt Technology) equipped with a laser operating at $\lambda=664 \mathrm{~nm}$. Degassed and filtered $(0.1 \mu \mathrm{m}$ membrane $) 0.15 \mathrm{M}$ ammonium acetate/0.20 $\mathrm{M}$ acetic acid buffer $(\mathrm{pH}=4.5)$ was used as eluent at a flow rate of $0.5 \mathrm{~mL} / \mathrm{min}$ (with a refractive index increment value, $\mathrm{dn} / \mathrm{dc}$ of $0.147 \mathrm{ml} / \mathrm{g}$ for dextran).These eluents were also used as solvent of samples, and the resulting solutions were filtered on a $0.45 \mu \mathrm{m}$ membrane before injection. Finally, $200 \mu \mathrm{L}$ of each sample at $1 \mathrm{mg} / \mathrm{mL}$ were injected. The data have been exploited thanks to the ASTRA 6.1.7.17 software (Wyatt Technology).

Cell culture. Murine macrophage cell line J774A.1 (ATCC) were grown in Dulbecco's Modified Eagle's Medium (Thermo Fischer) supplemented with 10\% v/v decomplemented fetal bovine serum (FBS) at $37^{\circ} \mathrm{C}$ in humidified conditions with 5\% CO . Prussian blue iron staining kit (Sigma-Aldrich) containing potassium ferrocyanide, pararosaniline and hydrochloric acid was used for cell staining..

\subsection{Synthesis and characterization of dextran-PEG}

2.2.1 Synthesis of 6-azidohexanoic acid (1): Compound 1 was prepared as described in literature $[23,24]$ with small modifications. Specifically, $\mathrm{NaN}_{3}(1 \mathrm{~g}, 15.4 \mathrm{mmol})$ was added to a solution of 6bromohexanoic acid $(1.5 \mathrm{~g}, 7.7 \mathrm{mmol})$ in dry DMF $(10 \mathrm{~mL})$ under $\mathrm{N}_{2}$ atmosphere and stirred at $85^{\circ} \mathrm{C}$ overnight until TLC (2:1 hexane:EtOAc) showed complete disappearance of the starting material and the appearance of a spot at $R_{\mathrm{f}}=0.59$. The solvent was rotary evaporated under high vacuum and the residue was dissolved in $\mathrm{H}_{2} \mathrm{O}(20 \mathrm{~mL})$ and extracted with EtOAc $(3 \times 30 \mathrm{~mL})$. The organic layers were combined, dried $\left(\mathrm{MgSO}_{4}\right)$, and rotary evaporated to give 6-azidohexanoic acid 1 (0.866 g, $5.5 \mathrm{mmol}$, $70 \%)$ as slightly yellow oil. NMR data agreed with those previously reported [24]: ${ }^{1} \mathrm{H} \mathrm{NMR}(300 \mathrm{MHz}$, $\left.\mathrm{CDCl}_{3}\right) \delta(\mathrm{ppm}): 11.04(\mathrm{~s}, 1 \mathrm{H}, \mathrm{COOH}), 3.24\left(\mathrm{t}, 2 \mathrm{H}, 3^{3}=6.9 \mathrm{~Hz}, \mathrm{CH}_{2} \mathrm{~N}_{3}\right), 2.32\left(\mathrm{t}, 2 \mathrm{H},{ }^{3} \mathrm{~J}=7.3 \mathrm{~Hz}\right.$, $\left.\mathrm{CH}_{2} \mathrm{COOH}\right), 1.70-1.50\left(\mathrm{~m}, 4 \mathrm{H}, \mathrm{CH}_{2} \mathrm{CH}_{2} \mathrm{COOH}, \mathrm{CH}_{2} \mathrm{CH}_{2} \mathrm{~N}_{3}\right), 1.40\left(\mathrm{~m}, 2 \mathrm{H}, \mathrm{CH}_{2} \mathrm{CH}_{2} \mathrm{CH}_{2} \mathrm{~N}_{3}\right) ;{ }^{13} \mathrm{C} \mathrm{NMR}(75$ $\left.\mathrm{MHz}_{2} \mathrm{CDCl}_{3}\right) \delta$ (ppm): $179.1(\mathrm{COOH}), 51.2\left(\mathrm{CH}_{2} \mathrm{~N}_{3}\right), 34.0\left(\mathrm{CH}_{2} \mathrm{COOH}\right), 28.6\left(\mathrm{CH}_{2} \mathrm{CH}_{2} \mathrm{~N}_{3}\right), 26.2$ $\left(\mathrm{CH}_{2} \mathrm{CH}_{2} \mathrm{CH}_{2} \mathrm{~N}_{3}\right), 24.3\left(\mathrm{CH}_{2} \mathrm{CH}_{2} \mathrm{COOH}\right)$.

2.2.2. Synthesis of 2,5-dioxopyrrolidin-1-yl 6-azidohexanoate (2): Compound 2 was prepared as described in literature [25] with small modifications. Specifically, $N$-hydroxysuccinimide $(520 \mathrm{mg}$, $4.5 \mathrm{mmol}$ ) was added to a solution of 6-azidohexanoic acid 1 (650 mg, $4.1 \mathrm{mmol})$ in dry $\mathrm{CH}_{2} \mathrm{Cl}_{2}(10$ $\mathrm{mL}$ ) under $\mathrm{N}_{2}$ atmosphere at room temperature and the mixture was stirred until complete solubilisation. Then, EDC (860 mg, $4.5 \mathrm{mmol}$ ) was added and the solution was stirred at room temperature until TLC (2:1 hexane:EtOAc) showed complete disappearance of the starting material and the appearance of a spot at $\mathrm{R}_{\mathrm{f}}=0.41$. After $16 \mathrm{~h}$, the mixture was washed with $1 \mathrm{~N} \mathrm{HCl}(2 \times 15 \mathrm{~mL})$ and saturated aq. $\mathrm{NaHCO}_{3}(2 \times 15 \mathrm{~mL})$. The aqueous layer was extracted with $\mathrm{CH}_{2} \mathrm{Cl}_{2}(2 \times 10 \mathrm{~mL})$. All organic phases were combined, dried $\left(\mathrm{MgSO}_{4}\right)$, and rotary evaporated. The residue was purified by column chromatography using 2:1 hexane:EtOAc as eluent to yield compound 2 (790 mg, $3.1 \mathrm{mmol}$, $76 \%)$ as a colourless liquid. NMR data agreed with those previously reported [25]: ${ }^{1} \mathrm{H} \mathrm{NMR}(300 \mathrm{MHz}$, $\left.\mathrm{CDCl}_{3}\right) \delta(\mathrm{ppm}): 3.23\left(\mathrm{t}, 2 \mathrm{H},{ }^{3} \mathrm{~J}=6.7 \mathrm{~Hz}, \mathrm{CH}_{2} \mathrm{~N}_{3}\right), 2.75\left(\mathrm{~s}, 4 \mathrm{H}, \mathrm{COCH}_{2} \mathrm{CH}_{2} \mathrm{CO}\right), 2.56\left(\mathrm{t}, 2 \mathrm{H}, 3^{3}=7.3 \mathrm{~Hz}\right.$, $\left.\mathrm{CH}_{2} \mathrm{COO}\right)$, $1.90-1.30\left(\mathrm{~m}, 6 \mathrm{H}, \mathrm{CH}_{2} \mathrm{CH}_{2} \mathrm{CH}_{2} \mathrm{CH}_{2} \mathrm{~N}_{3}\right) ;{ }^{13} \mathrm{C} \mathrm{NMR}\left(75 \mathrm{MHz}, \mathrm{CDCl}_{3}\right) \delta$ (ppm): 169.1 $\left(\mathrm{NCOCH}_{2}\right), 168.4(\mathrm{COO}), 51.1\left(\mathrm{CH}_{2} \mathrm{~N}_{3}\right), 30.8\left(\mathrm{CH}_{2} \mathrm{COO}\right), 28.4\left(\mathrm{CH}_{2}\right), 25.9\left(\mathrm{CH}_{2}\right), 25.6\left(\mathrm{COCH}_{2} \mathrm{CH}_{2} \mathrm{CO}\right)$, $24.1\left(\mathrm{CH}_{2}\right)$.

2.2.3. Synthesis of the sodium salt of [4-(6-azidohexanamido)-1-hydroxy-1 -(hydroxy-oxidophosphoryl)-butyl]phosphonic acid (3): Aqueous $0.1 \mathrm{M} \mathrm{NaOH} \mathrm{(} \mathrm{32} \mathrm{mL)} \mathrm{was} \mathrm{added} \mathrm{dropwise} \mathrm{to} \mathrm{a}$ suspension of alendronic acid monosodium salt trihydrate $(0.83 \mathrm{~g}, 2.56 \mathrm{mmol})$ in MilliQ water (18 $\mathrm{mL}$ ) until $\mathrm{pH} \sim 8.5$, forming a clear solution. A solution of 2,5-dioxopyrrolidin-1-yl 6-azidohexanoate $2(0.78 \mathrm{~g}, 3.1 \mathrm{mmol})$ in acetonitrile $(18 \mathrm{~mL})$ was added in four portions each $15 \mathrm{~min}$. Before each portion, $\mathrm{pH}$ was measured and readjusted to $\sim 8.5$ with aqueous $0.1 \mathrm{M} \mathrm{NaOH}$ if needed. The reaction mixture was stirred overnight at room temperature and then the solvent was rotary evaporated. The residue was purified by a short column chromatography using $5: 1 \rightarrow 2: 1 \mathrm{CH}_{3} \mathrm{CN}: \mathrm{H}_{2} \mathrm{O}$ as eluent to yield 
compound 3 (0.84 g, $2.05 \mathrm{mmol}, 80 \%)$ as a white solid after lyophilising: FT-IR (KBr) v/cm $\mathrm{cm}^{-1}: 3445$, 2938, 2867, 2100, 1632, 1558, 1105, 913, 620, 551; ${ }^{1} \mathrm{H} \mathrm{NMR}\left(600 \mathrm{MHz}, \mathrm{D}_{2} \mathrm{O}\right) \delta(\mathrm{ppm}): 3.34\left(\mathrm{t}, 2 \mathrm{H},{ }^{3} \mathrm{~J}=\right.$ $\left.6.9 \mathrm{~Hz}, \mathrm{CH}_{2} \mathrm{~N}_{3}\right), 3.22\left(\mathrm{t}, 2 \mathrm{H},{ }^{3} \mathrm{~J}=6.8 \mathrm{~Hz}, \mathrm{CONHCH}_{2}\right) 2.28\left(\mathrm{t}, 2 \mathrm{H}, 3 \mathrm{~J}=7.5 \mathrm{~Hz}, \mathrm{CH}_{2} \mathrm{CONH}\right), 2.01-1.93(\mathrm{~m}$, $\left.2 \mathrm{H}, \mathrm{CH}_{2} \mathrm{C}\right), 1.86-1.81\left(\mathrm{~m}, 2 \mathrm{H}, \mathrm{CH}_{2} \mathrm{CH}_{2} \mathrm{C}\right), 1.66-1.61\left(\mathrm{~m}, 4 \mathrm{H}, \mathrm{CH}_{2} \mathrm{CH}_{2} \mathrm{CONH}_{2} \mathrm{CH}_{2} \mathrm{CH}_{2} \mathrm{~N}_{3}\right), 1.42-1.37$ (m, $\left.2 \mathrm{H}, \mathrm{CH}_{2} \mathrm{CH}_{2} \mathrm{CH}_{2} \mathrm{~N}_{3}\right) ;{ }^{13} \mathrm{C} \mathrm{NMR}\left(150 \mathrm{MHz}, \mathrm{D}_{2} \mathrm{O}\right) \delta(\mathrm{ppm}): 176.9(\mathrm{CONH}), 73.9\left(\mathrm{t},{ }^{1} J_{\mathrm{CP}}=134.2 \mathrm{~Hz}\right.$, $\left.\mathrm{C}\left(\mathrm{PO}_{3}\right)_{2}\right), 51.0\left(\mathrm{CH}_{2} \mathrm{~N}_{3}\right), 40.0\left(\mathrm{NHCH}_{2}\right), 36.6\left(\mathrm{CH}_{2} \mathrm{CONH}\right), 31.1\left(\mathrm{CH}_{2} \mathrm{C}\right), 27.7\left(\mathrm{CH}_{2} \mathrm{CH}_{2} \mathrm{~N}_{3}\right), 25.4$ $\left(\mathrm{CH}_{2} \mathrm{CH}_{2} \mathrm{CH}_{2} \mathrm{~N}_{3}\right), 24.9\left(\mathrm{CH}_{2} \mathrm{CH}_{2} \mathrm{CONH}\right), 23.4\left(\mathrm{t},{ }^{3} \mathrm{CP}_{\mathrm{CP}}=5.9 \mathrm{~Hz}, \mathrm{CH}_{2} \mathrm{CH}_{2} \mathrm{C}\right) ;{ }^{31} \mathrm{P}\left(242.9 \mathrm{MHz}, \mathrm{D}_{2} \mathrm{O}\right) \delta(\mathrm{ppm})$ : 18.2 (C(PO3)2); [ESI-TOF-MS] $m / z$ calcd for $\mathrm{C}_{10} \mathrm{H}_{21} \mathrm{~N}_{4} \mathrm{O}_{8} \mathrm{P}_{2} 387.0840$, found 387.0839 [M - Na]; $\mathrm{m} / z$ calcd for $\mathrm{C}_{10} \mathrm{H}_{20} \mathrm{~N}_{4} \mathrm{O}_{8} \mathrm{P}_{2} \mathrm{Na} 409.0660$, found 409.0657 [M - H]; [ESI-TOF-MS] ${ }^{+} m / z$ calcd for $\mathrm{C}_{10} \mathrm{H}_{22} \mathrm{~N}_{4} \mathrm{O}_{8} \mathrm{P}_{2} \mathrm{Na}$ 411.0816 found $411.0818[\mathrm{M}+\mathrm{H}]^{+} ; m / z$ calcd for $\mathrm{C}_{10} \mathrm{H}_{21} \mathrm{~N}_{4} \mathrm{O}_{8} \mathrm{P}_{2} \mathrm{Na}_{2} 433.0635$, found $433.0643[\mathrm{M}+\mathrm{Na}]^{+}$; $m / z$ calcd for $\mathrm{C}_{10} \mathrm{H}_{20} \mathrm{~N}_{4} \mathrm{O}_{8} \mathrm{P}_{2} \mathrm{Na}_{3} 455.0455$ found $455.0465[\mathrm{M}-\mathrm{H}+2 \mathrm{Na}]^{+} ; m / z$ calcd for $\mathrm{C}_{10} \mathrm{H}_{19} \mathrm{~N}_{4} \mathrm{O}_{8} \mathrm{P}_{2} \mathrm{Na}_{4}$ 477.0274, found $477.0283[\mathrm{M}-2 \mathrm{H}+3 \mathrm{Na}]^{+}$.

2.2.4. Synthesis of 1-azido-1-deoxy- $\omega$-O-methoxypentatetracontaethylene glycol (4): A solution of MeOPEG ${ }_{45} \mathrm{OH}$ (35 g, $\left.17.375 \mathrm{mmol}\right)$, DMAP (428 mg, $\left.3.5 \mathrm{mmol}\right)$ and distilled Et $3 \mathrm{~N}(5.6 \mathrm{~mL}$, $40.250 \mathrm{mmol})$ in $\mathrm{CH}_{2} \mathrm{Cl}_{2}(40 \mathrm{~mL})$ was cooled to $0{ }^{\circ} \mathrm{C}$ under inert atmosphere. $\mathrm{MsCl}(2.7 \mathrm{~mL}, 35 \mathrm{mmol})$ was added dropwise over $15 \mathrm{~min}$ and the mixture was stirred at $0{ }^{\circ} \mathrm{C}$ during $30 \mathrm{~min}$ and then kept overnight at room temperature. The reaction mixture was then diluted with $\mathrm{CH}_{2} \mathrm{Cl}_{2}(50 \mathrm{~mL})$, and washed with $5 \% \mathrm{v} / \mathrm{v}$ aqueous $\mathrm{HCl}$ solution $(3 \times 50 \mathrm{~mL})$ and brine $(50 \mathrm{~mL})$. The organic phase was dried over $\mathrm{MgSO}_{4}$, filtered and concentrated under reduced pressure to dryness. The solid was subsequently dissolved in dry DMF $(40 \mathrm{~mL})$ and $\mathrm{NaN}_{3}(2.276 \mathrm{~g}, 35 \mathrm{mmol})$ was added. The mixture was stirred at $60^{\circ} \mathrm{C}$ for $24 \mathrm{~h}$ before the solvent was rotary evaporated under high vacuum. The residue was suspended in THF (20 mL) and sonicated (5 min), and filtered off. The clear organic filtrate was rotary evaporated, and the resulting solid was suspended in $\mathrm{Et}_{2} \mathrm{O}(50 \mathrm{~mL})$, sonicated $(5 \mathrm{~min})$ and filtered. The solid was dissolved in $\mathrm{H}_{2} \mathrm{O}(100 \mathrm{~mL})$ and extracted with $\mathrm{CH}_{2} \mathrm{Cl}_{2}(3 \times 100 \mathrm{~mL})$. The organic phases were combined, dried $\left(\mathrm{MgSO}_{4}\right)$ and rotary evaporated, and the residue dried under vacuum to give compound $4(26.225 \mathrm{~g}, 12.858 \mathrm{mmol}, 74 \%)$ as a slightly yellow powder: FT-IR $(\mathrm{KBr}) v / \mathrm{cm}^{-1}$ : 2868, 2105, 1093, 948, 842, 729; ${ }^{1} \mathrm{H}$ NMR (300 MHz, D $\left.{ }_{2} \mathrm{O}\right)$ 8: 3.96-3.93 (m, ${ }^{1}{ }_{\mathrm{H}, \mathrm{C}}=143.4 \mathrm{~Hz},{ }^{13} \mathrm{C}$ satellite peak), 3.75-3.68 (m, 176H, OCH $\left.\mathrm{CH}_{2} \mathrm{O}\right), 3.65-3.61\left(\mathrm{~m}, 2 \mathrm{H}, \mathrm{CH}_{2} \mathrm{CH}_{2} \mathrm{~N}_{3}\right), 3.53-3.49\left(\mathrm{~m}, 2 \mathrm{H}, \mathrm{CH}_{2} \mathrm{~N}_{3}\right)$, 3.49$3.46\left(\mathrm{~m},{ }^{1} \mathrm{~J}_{\mathrm{H}, \mathrm{C}}=71.7 \mathrm{~Hz},{ }^{13} \mathrm{C}\right.$ satellite peak), $3.38\left(\mathrm{~s}, \mathrm{CH}_{3} \mathrm{O}\right) ;{ }^{13} \mathrm{C} \mathrm{NMR}(75 \mathrm{MHz}, \mathrm{D} 2 \mathrm{O}) \delta: 71.7\left(\mathrm{MeOCH}_{2}\right)$, $70.3\left(\mathrm{OCH}_{2} \mathrm{CH}_{2} \mathrm{O}\right), 70.2\left(\mathrm{MeOCH}_{2} \mathrm{CH}_{2} \mathrm{O}\right), 70.0\left(\mathrm{OCH}_{2} \mathrm{CH}_{2} \mathrm{~N}_{3}\right), 58.8(\mathrm{OMe}), 50.9\left(\mathrm{CH}_{2} \mathrm{~N}_{3}\right)$.

2.2.5. Synthesis of dextran propargylcarbamate (DEX-PC, 5): $\mathrm{LiCl}$ (1 g) and dextran T-40 (4 g) were pre-dried at $80^{\circ} \mathrm{C}$ in a high vacuum oven for 2 days in the presence of $\mathrm{P}_{2} \mathrm{O}_{5}$. The mixture was further dried by suspension in dry toluene $(50 \mathrm{~mL})$ and subsequent vacuum distillation at $50{ }^{\circ} \mathrm{C}$. This azeotropic drying process with toluene was repeated twice, and then with anhydrous DMF (1x50 $\mathrm{mL}$ ) followed by evaporation of the solvent through a rotary evaporator at $60^{\circ} \mathrm{C}$ under high vacuum. The dry mixture was finally dissolved in anhydrous DMF $(80 \mathrm{~mL})$ and stirred at $80{ }^{\circ} \mathrm{C}$ for $1.5 \mathrm{~h}$. The solution was cooled down to room temperature before carbonyldiimidazole $(0.972 \mathrm{~g}, 0.006 \mathrm{mmol})$ was added, and stirred for $2.5 \mathrm{~h}$. Propargylamine $(3.85 \mathrm{~mL}, 0.06 \mathrm{mmol})$ was then added and the mixture stirred at room temperature for $24 \mathrm{~h}$. The solvent volume was reduced to $60 \mathrm{~mL}$ by rotary evaporation and the solution was poured into isopropanol $(0.9 \mathrm{~L})$. The resulting pale yellow solid was collected by filtration and dissolved in $\mathrm{H}_{2} \mathrm{O}(100 \mathrm{~mL})$, syringe filtered $(0.45 \mu \mathrm{m})$ and dialyzed (12000-14000 Da MWCO) against distilled water, changing dialysate solution each 3 hours until its conductivity was stable and below $1 \mu \mathrm{S} / \mathrm{cm}$ (3 days for final value of $0.90 \mu \mathrm{S} / \mathrm{cm}$ ) to yield DEX-PC 5 (3.6 g) as a white solid after lyophilization: FT-IR (KBr) v/ $\mathrm{cm}^{-1}: 3420,2930,1709,1639,1530,1461,1419$, 1346, 1264, 1156, 1041, 1014, 766, 549, 527; ${ }^{1} \mathrm{H}$ NMR (600 MHz, D2O) $\delta(p p m): 5.36\left(\mathrm{~d}, 3_{1,2}^{3}=3.8 \mathrm{~Hz}\right.$, $\alpha(1 \rightarrow 3,4) \mathrm{H}-1), 5.21\left(\mathrm{app} b d, J_{\mathrm{app}}=2.8 \mathrm{~Hz}, \alpha\right.$ reducing end $\left.\mathrm{H}-1, \mathrm{CH}-\mathrm{OCONH}\right), 5.06\left(\mathrm{~d}, 3 \mathrm{~J}_{1,2}=2.6 \mathrm{~Hz}, \mathrm{H}-\right.$ $\left.1^{\mathrm{s}}\right), 5.01\left(\mathrm{~d}, 3 \mathrm{~J}_{1,2}=3.0 \mathrm{~Hz}, \alpha(1 \rightarrow 6) \mathrm{H}-1\right), 4.98\left(\mathrm{bs}, \mathrm{H}-1^{\mathrm{s}}\right), 4.63\left(\mathrm{~d}, 3 \mathrm{~J}_{1,2}=7.2 \mathrm{~Hz}, \beta\right.$ reducing end H-1), 4.02$3.93\left(\mathrm{~m}, \mathrm{H}-3,6^{\mathrm{a}}, \mathrm{CH}_{2} \mathrm{C} \equiv\right), 3.78-3.73\left(\mathrm{~m}, \mathrm{H}-5,6^{\mathrm{b}}\right), 3.60\left(\mathrm{dd}, 3_{1,2}=3.0 \mathrm{~Hz}, 3 J_{2,3}=9.7 \mathrm{~Hz}, \mathrm{H}-2\right), 3.55(\mathrm{t}, 3 \mathrm{~J}=9.4$

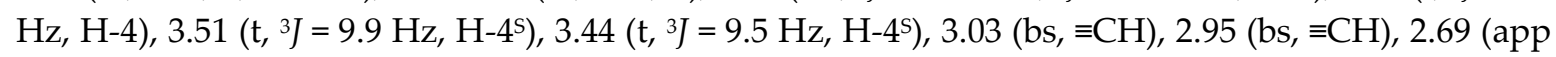
bd, $\left.J_{\text {app }}=8.7 \mathrm{~Hz}, \equiv \mathrm{CH}\right) ;{ }^{13} \mathrm{C} \mathrm{NMR}\left(150 \mathrm{MHz}, \mathrm{D}_{2} \mathrm{O}\right) \delta$ (ppm): $157.9(\mathrm{CO}), 157.0(\mathrm{CO}), 99.3,98.2,97.7$ $(\alpha(1 \rightarrow 6) \mathrm{C}-1), 95.6\left(\mathrm{C}-1^{\mathrm{S}}\right), 80.4-78.8(\mathrm{C} \equiv), 76.7,73.4(\mathrm{C}-3), 73.2-73.1(\equiv \mathrm{CH}), 72.0,71.4(\mathrm{C}-2), 71.0,70.2(\mathrm{C}-$ 
5), 69.9, 69.5 (C-4), 67.7, 65.5 (C-6), 65.2, 60.5, 30.1 ( $\left.\mathrm{CH}_{2} \mathrm{C} \equiv\right)$; Anal. found C 40.90\%, H 6.536\%, N 1.42\%. The number average molecular weight and the molar-mass dispersity were measured to be $M n=$ $35,240 \mathrm{~g} / \mathrm{mol}$ and $\mathrm{Ð}=1.14$, respectively.

2.2.6. Synthesis of DEX-ALN75-PEG 25 (6): Pre-dried LiCl (90 mg, $2.123 \mathrm{mmol}$ ) and DEX-PC 5 (350 $\mathrm{mg}, 0.0079 \mathrm{mmol}$ containing $0.397 \mathrm{mmol}$ of propargyl groups) were dissolved in $\mathrm{H}_{2} \mathrm{O}(5 \mathrm{~mL})$ and heated at $60^{\circ} \mathrm{C}$. A solution of sodium alendronate derivative $3(127 \mathrm{mg}, 0.298 \mathrm{mmol})$ in $\mathrm{H}_{2} \mathrm{O}(2 \mathrm{~mL})$ was added, followed by a suspension of $\mathrm{CuSO}_{4}(29 \mathrm{mg}, 0.179 \mathrm{mmol})$ and sodium ascorbate $(118 \mathrm{mg}$, $0.596 \mathrm{mmol})$ in $\mathrm{H}_{2} \mathrm{O}(1 \mathrm{~mL})$, and the mixture was stirred at $90^{\circ} \mathrm{C}$ overnight. After cooling down to room temperature, azide $4(809 \mathrm{mg}, 0.397 \mathrm{mmol})$ and a suspension of $\mathrm{CuSO}_{4}(38 \mathrm{mg}, 0.238 \mathrm{mmol})$ and sodium ascorbate $(157 \mathrm{mg}, 0.794 \mathrm{mmol})$ in $\mathrm{H}_{2} \mathrm{O}(0.8 \mathrm{~mL})$ were subsequently added. The mixture was stirred for $22 \mathrm{~h}$ at $90{ }^{\circ} \mathrm{C}$, then diluted with $\mathrm{H}_{2} \mathrm{O}(10 \mathrm{~mL})$, filtered through filtering paper and then through a $0.45 \mu \mathrm{m}$ syringe filter. Aqueous $0.1 \mathrm{M} \mathrm{NaOH}$ was added until $\mathrm{pH} \sim 7.0$, followed by a solution of $10 \mathrm{mM}$ EDTA at pH $7.0(20 \mathrm{~mL})$. The $\mathrm{pH}$ was then monitored and kept at $\sim 7.0$ with $0.1 \mathrm{M}$ $\mathrm{NaOH}$ for $24 \mathrm{~h}$. The mixture was again filtered through $0.45 \mu \mathrm{m}$ syringe filter, lyophilized, redissolved in the minimum amount of water and dialyzed (12000-14000 Da MWCO) against distilled water, changing dialysate solution each 3 hours until its conductivity was stable and below $1 \mu \mathrm{S} / \mathrm{cm}$ (3 days for final value of $0.60 \mu \mathrm{S} / \mathrm{cm}$ ) to yield DEX-ALN75-PEG25 $7(881 \mathrm{mg}$ ) as a brownish solid after lyophilization: FT-IR (KBr) v/cm-1: 3423, 2913, 2880, 1645, 1457, 1352, 1298, 1252, 1104, 1041, 952, 845, 548; ${ }^{1} \mathrm{H}$ NMR (600 MHz, $\left.\mathrm{D}_{2} \mathrm{O}\right) \delta(\mathrm{ppm}): 8.03$ (bs, H-5- $\left.\mathrm{C}_{2} \mathrm{HN}_{3}\right), 5.35$ (bs, $\left.\alpha(1 \rightarrow 3,4) \mathrm{H}-1\right), 5.27$ (bs, $\alpha$ reducing end H-1), 5.18 (app t, Japp $=5.0 \mathrm{~Hz}, \mathrm{CH}-\mathrm{OCONH}), 5.04\left(\mathrm{bs}, \mathrm{H}-1^{\mathrm{s}}\right), 5.00(\mathrm{bs}, \alpha(1 \rightarrow 6) \mathrm{H}-1), 4.64$ (bs, $\beta$ reducing end $\mathrm{H}-1), 4.51-4.44\left(\mathrm{~m}, \mathrm{OCH}_{2} \mathrm{CH}_{2}-\mathrm{C}_{2} \mathrm{HN}_{3}\right), 4.40-4.38\left(\mathrm{~m}, \mathrm{CH}_{2}-\mathrm{C}_{2} \mathrm{HN}_{3}\right), 4.31-4.28(\mathrm{~m}$, $\left.\mathrm{NHCH}_{2}-\mathrm{C}_{2} \mathrm{HN}_{3}\right), 4.02-3.93(\mathrm{~m}, \mathrm{H}-5,6 \mathrm{6}), 3.87-3.84\left(\mathrm{~m},{ }^{1}{ }_{\mathrm{H}, \mathrm{C}}=140.2 \mathrm{~Hz},{ }^{13} \mathrm{C}\right.$ satellite peak), 3.73 (bs, $\mathrm{H}-3,66^{\mathrm{b}}$, $\mathrm{OCH}_{2} \mathrm{CH}_{2} \mathrm{O}$ ), 3.67-3.64 (m, $\left.\mathrm{OCH}_{2} \mathrm{CH}_{2}-\mathrm{C}_{2} \mathrm{HN}_{3}\right), 3.61-3.60(\mathrm{~m}, \mathrm{H}-2), 3.56-3.52(\mathrm{~m}, \mathrm{H}-4), 3.45$ (app t, Japp = $9.3 \mathrm{~Hz}, \mathrm{H}-4 \mathrm{~s}), 3.40\left(\mathrm{~s}, \mathrm{CH}_{3} \mathrm{O}\right), 3.34-3.19\left(\mathrm{CH}_{2} \mathrm{NH}\right), 2.88(\mathrm{bs}, \equiv \mathrm{CH}), 2.78(\mathrm{bs}, \equiv \mathrm{CH}), 2.64(\mathrm{bs}, \equiv \mathrm{CH}), 2.23$ (bs, $\mathrm{CH}_{2} \mathrm{CO}$ ), 1.93 (bs, $\mathrm{CH}_{2} \mathrm{C}$ ), 1.77 (bs, $\left.\mathrm{CH}_{2} \mathrm{CH}_{2} \mathrm{C}\right), 1.61\left(\mathrm{CH}_{2} \mathrm{CH}_{2} \mathrm{CO}, \mathrm{CH}_{2} \mathrm{CH}_{2}-\mathrm{C}_{2} \mathrm{HN}_{3}\right), 1.30-1.16$ (bs, $\left.\mathrm{CH}_{2} \mathrm{CH}_{2} \mathrm{CH}_{2} \mathrm{CO}\right)$; ${ }^{31} \mathrm{P}$ NMR $\left(242.9 \mathrm{MHz}, \mathrm{D}_{2} \mathrm{O}\right) \delta(\mathrm{ppm})$ : -1.4-(-2.6) $\left.\left(\mathrm{C}_{(\mathrm{PO}}\right)_{2}\right)$; Anal.: found $\mathrm{C} 46.24 \%, \mathrm{H}$ $7.582 \%$, N 2.46\%. HR-ICP-MS: found P $0.81 \%$, Na $0.103 \%$, Cu 1.05\%, Li $0.042 \%$.

2.2.7. Synthesis of DEX-ALN50-PEG 50 (7): Pre-dried LiCl (90 mg, $2.123 \mathrm{mmol}$ ) and DEX-PC 5 (350 $\mathrm{mg}, 0.0079 \mathrm{mmol}$ containing $0.397 \mathrm{mmol}$ of propargyl groups) were dissolved in $\mathrm{H}_{2} \mathrm{O}(5 \mathrm{~mL})$ and heated at $60^{\circ} \mathrm{C}$. A solution of sodium alendronate derivative $3(85 \mathrm{mg}, 0.198 \mathrm{mmol})$ in $\mathrm{H}_{2} \mathrm{O}(1.5 \mathrm{~mL})$ was added, followed by a suspension of $\mathrm{CuSO}_{4}(19 \mathrm{mg}, 0.119 \mathrm{mmol})$ and sodium ascorbate $(79 \mathrm{mg}$, $0.397 \mathrm{mmol})$ in $\mathrm{H}_{2} \mathrm{O}(1 \mathrm{~mL})$, and the mixture was stirred at $90^{\circ} \mathrm{C}$ overnight. After cooling down to room temperature, azide $4(809 \mathrm{mg}, 0.397 \mathrm{mmol})$ and a suspension of $\mathrm{CuSO}_{4}(38 \mathrm{mg}, 0.238 \mathrm{mmol})$ and sodium ascorbate $(157 \mathrm{mg}, 0.794 \mathrm{mmol})$ in $\mathrm{H}_{2} \mathrm{O}(0.8 \mathrm{~mL})$ were subsequently added. The mixture was stirred for $22 \mathrm{~h}$ at $90{ }^{\circ} \mathrm{C}$, then diluted with $\mathrm{H}_{2} \mathrm{O}(10 \mathrm{~mL})$, filtered through filtering paper and then through a $0.45 \mu \mathrm{m}$ syringe filter. Aqueous $0.1 \mathrm{M} \mathrm{NaOH}$ was added until $\mathrm{pH} \sim 7.0$, followed by a solution of $10 \mathrm{mM}$ EDTA at $\mathrm{pH} 7.0(20 \mathrm{~mL})$. The $\mathrm{pH}$ was then monitored and kept at $\sim 7.0$ with $0.1 \mathrm{M}$ $\mathrm{NaOH}$ for $24 \mathrm{~h}$. The mixture was again filtered through $0.45 \mu \mathrm{m}$ syringe filter, lyophilized, redissolved in the minimum amount of water and dialyzed (12000-14000 Da MWCO) against distilled water, changing dialysate solution each 3 hours until its conductivity was stable and below $1 \mu \mathrm{S} / \mathrm{cm}$ ( 3 days for final value of $0.74 \mu \mathrm{S} / \mathrm{cm}$ ) to yield DEX-ALN50-PEG50 $6(928 \mathrm{mg}$ ) as a brownish solid after lyophilization: FT-IR (KBr) v/cm ${ }^{-1}: 3429,2917,2881,1645,1457,1352,1253,1103,952,558 ;{ }^{1} \mathrm{H}$ NMR $\left(600 \mathrm{MHz}, \mathrm{D}_{2} \mathrm{O}\right) \delta$ (ppm): 8.03 (bs, H-5- $\left.\mathrm{C}_{2} \mathrm{HN}_{3}\right), 5.35$ (bs, $\left.\alpha(1 \rightarrow 3,4) \mathrm{H}-1\right), 5.26$ (bs, $\alpha$ reducing end H1), $5.18\left(\mathrm{app} \mathrm{t}, J_{\mathrm{app}}=5.0 \mathrm{~Hz}, \mathrm{CH}-\mathrm{OCONH}\right), 5.04\left(\mathrm{bs}, \mathrm{H}-1^{\mathrm{s}}\right), 5.00(\mathrm{bs}, \alpha(1 \rightarrow 6) \mathrm{H}-1), 4.64$ (bs, $\beta$ reducing end $\mathrm{H}-1)$, 4.47-4.43 (m, $\left.\mathrm{OCH}_{2} \mathrm{CH}_{2}-\mathrm{C}_{2} \mathrm{HN}_{3}\right), 4.40-4.38\left(\mathrm{~m}, \mathrm{CH}_{2}-\mathrm{C}_{2} \mathrm{HN}_{3}\right), 4.31-4.29\left(\mathrm{~m}, \mathrm{NHCH}_{2}-\mathrm{C}_{2} \mathrm{HN}_{3}\right)$, 4.02-3.93 (m, H-5,6a), 3.87-3.84 (m, ${ }^{1} \mathrm{JH}_{\mathrm{H}} \mathrm{C}=140.4 \mathrm{~Hz},{ }^{13} \mathrm{C}$ satellite peak), 3.73 (bs, $\left.\mathrm{H}-3,6^{\mathrm{b}}, \mathrm{OCH}_{2} \mathrm{CH}_{2} \mathrm{O}\right)$, 3.67-3.64 (m, $\left.\mathrm{OCH}_{2} \mathrm{CH}_{2}-\mathrm{C}_{2} \mathrm{HN}_{3}\right), 3.61-3.59(\mathrm{~m}, \mathrm{H}-2), 3.56-3.52(\mathrm{~m}, \mathrm{H}-4), 3.43$ (app t, Japp $=10.0 \mathrm{~Hz}, \mathrm{H}-$ $\left.4^{\mathrm{s}}\right), 3.41\left(\mathrm{~s}, \mathrm{CH}_{3} \mathrm{O}\right), 3.32-3.18\left(\mathrm{CH}_{2} \mathrm{NH}\right), 2.88(\mathrm{bs}, \equiv \mathrm{CH}), 2.70(\mathrm{bs}, \equiv \mathrm{CH}), 2.25$ (bs, $\left.\mathrm{CH}_{2} \mathrm{CO}\right), 1.93$ (bs, $\mathrm{CH}_{2} \mathrm{C}$ ), 1.78 (bs, $\left.\mathrm{CH}_{2} \mathrm{CH}_{2} \mathrm{C}\right), 1.61\left(\mathrm{CH}_{2} \mathrm{CH}_{2} \mathrm{CO}, \mathrm{CH}_{2} \mathrm{CH}_{2}-\mathrm{C}_{2} \mathrm{HN}_{3}\right), 1.26$ (bs, $\left.\mathrm{CH}_{2} \mathrm{CH}_{2} \mathrm{CH}_{2} \mathrm{CO}\right) ;{ }^{31} \mathrm{P} \mathrm{NMR}$ (242.9 MHz, $\left.\mathrm{D}_{2} \mathrm{O}\right) \delta$ (ppm): -1.8-(-2.9) (C(PO3)2); Anal.: found C 46.85\%, H 7.769\%, N 2.27\%. HR-ICP- 
MS: found P $0.57 \%$, Na $0.060 \%, \mathrm{Cu} 0.74 \%$, Li $0.027 \%$. The number average molecular weight and the molar-mass dispersity were measured to be $M n=83,120 \mathrm{~g} / \mathrm{mol}$ and $Đ=1.36$, respectively.

2.2.8. Synthesis of DEX-ALN25-PEG75 (8): Pre-dried LiCl (90 mg, $2.123 \mathrm{mmol}$ ) and DEX-PC 5 (350 $\mathrm{mg}, 0.0079 \mathrm{mmol}$ containing $0.397 \mathrm{mmol}$ of propargyl groups) were dissolved in $\mathrm{H}_{2} \mathrm{O}(5 \mathrm{~mL})$ and heated at $60^{\circ} \mathrm{C}$. A solution of sodium alendronate derivative $3(43 \mathrm{mg}, 0.100 \mathrm{mmol})$ in $\mathrm{H}_{2} \mathrm{O}(1 \mathrm{~mL})$ was added, followed by a suspension of $\mathrm{CuSO}_{4}(10 \mathrm{mg}, 0.060 \mathrm{mmol})$ and sodium ascorbate $(40 \mathrm{mg}$, $0.200 \mathrm{mmol})$ in $\mathrm{H}_{2} \mathrm{O}(1 \mathrm{~mL})$, and the mixture was stirred at $90^{\circ} \mathrm{C}$ overnight. After cooling down to room temperature, azide $4(809 \mathrm{mg}, 0.397 \mathrm{mmol})$ and a suspension of $\mathrm{CuSO}_{4}(38 \mathrm{mg}, 0.238 \mathrm{mmol})$ and sodium ascorbate $(157 \mathrm{mg}, 0.794 \mathrm{mmol})$ in $\mathrm{H}_{2} \mathrm{O}(0.8 \mathrm{~mL})$ were subsequently added. The mixture was stirred for $22 \mathrm{~h}$ at $90{ }^{\circ} \mathrm{C}$, then diluted with $\mathrm{H}_{2} \mathrm{O}(10 \mathrm{~mL})$, filtered through filtering paper and then through a $0.45 \mu \mathrm{m}$ syringe filter. Aqueous $0.1 \mathrm{M} \mathrm{NaOH}$ was added until $\mathrm{pH} \sim 7.0$, followed by a solution of $10 \mathrm{mM}$ EDTA at $\mathrm{pH} 7.0(20 \mathrm{~mL})$. $\mathrm{pH}$ was then monitored and kept at $\sim 7.0$ with $0.1 \mathrm{M}$ $\mathrm{NaOH}$ for $24 \mathrm{~h}$. The mixture was again filtered through $0.45 \mu \mathrm{m}$ syringe filter, lyophilized, redissolved in the minimum amount of water and dialyzed (12000-14000 Da MWCO) against distilled water, changing dialysate solution each 3 hours until its conductivity was stable and below $1 \mu \mathrm{S} / \mathrm{cm}$ (2 days for final value of $0.63 \mu \mathrm{S} / \mathrm{cm}$ ) to yield DEX-ALN25-PEG75 $8(978 \mathrm{mg}$ ) as a brownish solid after lyophilization: FT-IR (KBr) v/cm-1: 3423, 2912, 2880, 1645, 1457, 1352, 1299, 1253, 1104, 1042, 1019, 951, 845, 550; ${ }^{1} \mathrm{H}$ NMR (600 MHz, D2O) $\delta$ (ppm): 8.03 (bs, H-5-C2 $\mathrm{HN}_{3}$ ), 5.35 (bs, $\left.\alpha(1 \rightarrow 3,4) \mathrm{H}-1\right), 5.27$ (bs, $\alpha$ reducing end H-1), 5.19 (app t, Japp $=5.0 \mathrm{~Hz}, \mathrm{CH}-\mathrm{OCONH}), 5.05\left(\mathrm{bs}, \mathrm{H}-1^{\mathrm{s}}\right), 5.00(\mathrm{bs}, \alpha(1 \rightarrow 6) \mathrm{H}-1), 4.90$ (bs, $\left.\mathrm{H}-1^{\mathrm{s}}\right), 4.64$ (bs, $\beta$ reducing end $\left.\mathrm{H}-1\right), 4.64\left(\mathrm{bs}, \mathrm{OCH}_{2} \mathrm{CH}_{2}-\mathrm{C}_{2} \mathrm{HN}_{3}\right), 4.40-4.38\left(\mathrm{~m}, \mathrm{CH}_{2}-\mathrm{C}_{2} \mathrm{HN}_{3}\right), 4.31-$ $4.28\left(\mathrm{~m}, \mathrm{NHCH}_{2}-\mathrm{C}_{2} \mathrm{HN}_{3}\right), 4.02-3.93(\mathrm{~m}, \mathrm{H}-5,6 \mathrm{6}), 3.85-3.84\left(\mathrm{~m},{ }^{1} \mathrm{~J}_{\mathrm{H}, \mathrm{C}}=140.4 \mathrm{~Hz},{ }^{13} \mathrm{C}\right.$ satellite peak), 3.73 (bs, $\left.\mathrm{H}-3,6^{\mathrm{b}}, \mathrm{OCH}_{2} \mathrm{CH}_{2} \mathrm{O}\right), 3.68-3.64\left(\mathrm{~m}, \mathrm{OCH}_{2} \mathrm{CH}_{2}-\mathrm{C}_{2} \mathrm{HN}_{3}\right), 3.61-3.59(\mathrm{~m}, \mathrm{H}-2), 3.56-3.52(\mathrm{~m}, \mathrm{H}-4), 3.45$ (app t, Japp = 9.6 Hz, H-4 ${ }^{\mathrm{s}}$ ), $3.41\left(\mathrm{~s}, \mathrm{CH}_{3} \mathrm{O}\right), 3.32-3.19\left(\mathrm{CH}_{2} \mathrm{NH}\right), 3.02(\mathrm{bs}, \equiv \mathrm{CH}), 2.95$ (bs, $\left.\equiv \mathrm{CH}\right), 2.23$ (bs, $\mathrm{CH}_{2} \mathrm{CO}$ ), 1.93 (bs, $\mathrm{CH}_{2} \mathrm{C}$ ), 1.78 (bs, $\mathrm{CH}_{2} \mathrm{CH}_{2} \mathrm{C}$ ), $1.61\left(\mathrm{CH}_{2} \mathrm{CH}_{2} \mathrm{CO}, \mathrm{CH}_{2} \mathrm{CH}_{2}-\mathrm{C}_{2} \mathrm{HN}_{3}\right), 1.31-1.22$ (bs, $\mathrm{CH}_{2} \mathrm{CH}_{2} \mathrm{CH}_{2} \mathrm{CO}$ ); ${ }^{31} \mathrm{P}$ NMR (242.9 MHz, $\left.\mathrm{D}_{2} \mathrm{O}\right) \delta$ (ppm): 0.5-0.1, -1.5-(-2.4) $\left(\mathrm{C}\left(\mathrm{PO}_{3}\right)_{2}\right)$; Anal.: found $\mathrm{C}$ $47.86 \%$, H 7.842\%, N 2.05\%. HR-ICP-MS: found P 0.26\%, Na 0.092\%, Cu 0.42\%, Li 0.048\%.

\subsection{Synthesis and characterization of MIL-100(Fe) nanoMOFs}

Iron trimesate nanoMOFs were synthesized using a microwave-assisted hydrothermal method as previously described [6]. Briefly, $30 \mathrm{~mL}$ of an aqueous mixture containing $6.0 \mathrm{mM}$ iron chloride hexahydrate and $4.0 \mathrm{mM}$ of 1,3,5-benzenetricarboxylic acid was heated at $130^{\circ} \mathrm{C}$ under stirring prior to microwave irradiation at $1600 \mathrm{~W}$ (Mars-5, CEM, USA). The synthesized nanoMOFs were recovered by centrifugation at $10000 \mathrm{~g}$ for $15 \mathrm{~min}$ and purified by washing six times with absolute ethanol. Their morphology was observed with a transmission electron microscope (TEM, JEOL $1400(120 \mathrm{kV})$, Japan). Mean hydrodynamic diameters and size distributions were determined by dynamic light scattering (DLS, Malvern Nano-ZS, Zetasizer Nano series, France). NanoMOFs' Zeta potential (ZP) was measured at $25{ }^{\circ} \mathrm{C}$ using a Zetasizer Nano-ZS instrument in a $\mathrm{pH}$ range of 1 to 10 . For this, nanoMOFs were diluted to a final concentration of $100 \mu \mathrm{g} / \mathrm{mL}$ using a $1 \mathrm{mM} \mathrm{KCl}$ solution. The nanoMOF specific surface area was measured by nitrogen sorption experiments at $-196^{\circ} \mathrm{C}$ on an ASAP 2020 (Micromeritics, USA) after sample degassing at $100{ }^{\circ} \mathrm{C}$ for $15 \mathrm{~h}$ under high vacuum. Xray powder diffraction patterns (XRPD) were recorded for crystallinity characterization. NanoMOFs were stored in ethanol at room temperature and re-suspended in aqueous media whenever needed.

\subsection{Surface modification of MIL-100(Fe) nanoMOFs and their characterization}

\subsubsection{Preparation and characterization of DEX-ALN-PEG coated nanoMOF}

NanoMOFs were centrifuged at $10000 \mathrm{~g}$ for $10 \mathrm{~min}$ to remove the storage solvent (ethanol) and then re-dispersed in water by vortex. For coating, they were incubated overnight at room temperature with DEX-ALN-PEG solutions at mass ratios DEX-ALN-PEG: nanoMOFs of 1:4, 1:2 and 1:1. The nonattached DEX-ALN-PEG fraction was removed by centrifugation (10000 g, $10 \mathrm{~min})$. The pellets were 
dried and the adsorbed DEX-ALN-PEG was quantified by inductively coupled plasma mass spectrometry (ICP-MS). Briefly, nanoMOFs before and after modification with DEX-ALN-PEG were digested using aqua regia (15 minutes under ultrasonic bath), and phosphorous ( $\mathrm{P}$ ) quantification was performed using an ICP-MS equipped with a triple quadrupole (Agilent 8800, Agilent Technologies, Japan). Operation conditions were daily optimized using a tuning solution. P isotope was detected using "mass shift mode" $\left.{ }^{(47} \mathrm{PO}^{+}\right)$after reaction with oxygen in the cell. Conversely, scandium (Sc) (added as internal standard on samples and calibration standards solutions at a concentration of 10 $\mu \mathrm{g} / \mathrm{L}$ ) was detected on "mass mode" $\left.{ }^{45} \mathrm{Sc}^{+}\right)$. Oxygen was introduced into the collision/reaction cell at a flow rate of $0.35 \mathrm{~mL} / \mathrm{min}$. Dwell time for each of the targeted isotopes was $1 \mathrm{~s}$. P was quantified using external calibration using certified $1000 \mathrm{mg} / \mathrm{L}$ P standard solution (Merck, Germany).

The amount A of DEX-ALN-PEG associated to nanoMOFs was calculated on the basis of their P content by the following formula:

$$
A=\frac{P 1}{P 2} 100 \%
$$

where (P1 $\mathrm{wt} \%$ ) is the phosphorous content in the coated nanoMOFs and (P2 $\mathrm{wt} \%) \%$ ) is the phosphorous content in the synthesized DEX-ALN-PEG copolymers.

DEX-ALN-PEG coated nanoMOF were characterized in the same fashion as the uncoated samples to determine their size distribution, morphology and surface charge. The crystallinity of DEX-ALN-PEG coated nanoMOF was characterized by XRPD.

\subsubsection{Colloidal stability investigation}

Colloidal stabilities of DEX-ALN-PEG coated nanoMOFs were estimated by DLS after incubation in both MilliQ water and cell culture medium (DMEM complemented with 10\% FBS, 1\% penicillin/streptomycin $(100 \mathrm{mg} / \mathrm{mL})$ and $1 \%$ L-glutamine). Mean hydrodynamic diameters were measured at $6 \mathrm{~h}, 1,2,4,7,9,14,18$ and $21 \mathrm{~d}$ storage at $4{ }^{\circ} \mathrm{C}$ in water, and at $0,2,4,6$, and $8 \mathrm{~h}$ after incubation at $37^{\circ} \mathrm{C}$ in cell culture media.

\subsubsection{Human albumin adsorption studies}

NanoMOFs coated or not with DEX-ALN-PEG $(300 \mu \mathrm{g} / \mathrm{mL})$ were incubated with HSA solutions at $100 \mu \mathrm{g} / \mathrm{mL}$ in $10 \mathrm{mM}$ phosphate buffer (PB) at $37^{\circ} \mathrm{C}$. The samples were centrifuged at $10000 \mathrm{~g}$ for $5 \mathrm{~min}$ to recover the nanoMOF pellets after $1,2,3,4,6,8$ and $12 \mathrm{~h}$ incubation. The excessive HSA in the supernatant was quantified using a bicinchoninic acid (BCA) assay.

\subsection{4 "Stealth" effect of the DEX-ALN-PEG shell}

Visualization by optical microscopy. A direct visualization of the MIL-100(Fe) nanoMOFs inside J774A.1 macrophages was achieved based on iron staining using a Prussian blue staining kit. In a 24-well plate (with cover slips) $1 \times 10^{5}$ J774A.1 macrophages were placed with RPMI complete medium and $10 \%$ FBS. Each well of macrophages was treated with $50 \mu \mathrm{g}$ of nanoMOFs, either coated or not with DEX-ALN-PEG. The macrophages were subsequently incubated for 2 and $4 \mathrm{~h}$ in an incubator at $37^{\circ} \mathrm{C}$ with a $\mathrm{CO}_{2}$ concentration of $5 \%$. Afterwards the wells were washed with complete medium and then further washed twice with PBS. Cells were fixed with $4 \%$ paraformaldehyde, washed and incubated for 10 minutes with $2 \%$ potassium ferrocyanide in $0.6 \mathrm{mM}$ hydrochloric acid. Cells were washed again with PBS, counterstained with pararosaniline hydrochloride $(0.02 \%)$ and placed on a glass slide. A drop of emersion oil was applied to each coverslip and evaluated for iron staining using light microscopy.

Fe quantification by ICP-MS. Macrophage cells (J774A.1) were seeded overnight at a density of $3.0 \times 10^{5}$ cells per well in 24 -well plates in cell culture medium at $37^{\circ} \mathrm{C}$ in $5 \% \mathrm{CO}_{2}$. Cells were then incubated with $1 \mathrm{~mL}$ cell culture media containing nanoMOFs coated or not with DEX-ALN-PEG for 2,4 and $6 \mathrm{~h}$ (nanoMOFs concentration $=50 \mu \mathrm{g} / \mathrm{mL}$ ). At the end of the incubation, the cells were 
washed with PBS for three times to eliminate the nanoMOFs which did not interact with the cells. Cells were finally dried and digested using aqua regia (15 minutes under ultrasonic bath). Fe quantification was performed using an ICP-MS equipped with a triple quadrupole (Agilent 8800). Operation conditions were daily optimized using a tuning solution. Fe and Co (added as internal standard on samples and calibration standards solution at a concentration of $10 \mu \mathrm{g} / \mathrm{L}$ ) isotopes were detected using "on-mass mode" $\left({ }^{54} \mathrm{Fe}^{+},{ }^{56} \mathrm{Fe}^{+},{ }^{59} \mathrm{Co}^{+}\right)$. Helium was introduced into the collision/reaction cell at a flow rate of $3 \mathrm{~mL} \mathrm{~min}^{-1}$. Dwell time for each of the targeted isotopes was $1 \mathrm{~s}$. Fe was quantified using external calibration prepared using certified $1000 \mathrm{mg} / \mathrm{L}$ Fe standard solution (Merck, Germany).

\section{Results and discussion}

\subsection{Synthesis and characterization of DEX-ALN-PEG copolymers}

For the post-synthetic modification of the surface of MIL-100(Fe) MOF nanoparticles we planned to prepare DEX derivatives appended with alendronate (ALN) moieties and PEG chains. For this strategy, we first prepared alendronate and PEG azide derivatives 3 and $\mathbf{4}$, respectively (Scheme $\mathbf{1}$ ). We then appended them on dextran T-40 grafted with a controlled number of therminal alkyne residues in the form of propargyl carbamate groups (DEX-PC 5) (Scheme 2) through a $\mathrm{Cu}(\mathrm{I})$-catalyzed azide-alkyne cycloaddition (CuAAC) reaction to yield DEX-ALN-PEG conjugates 6-8.
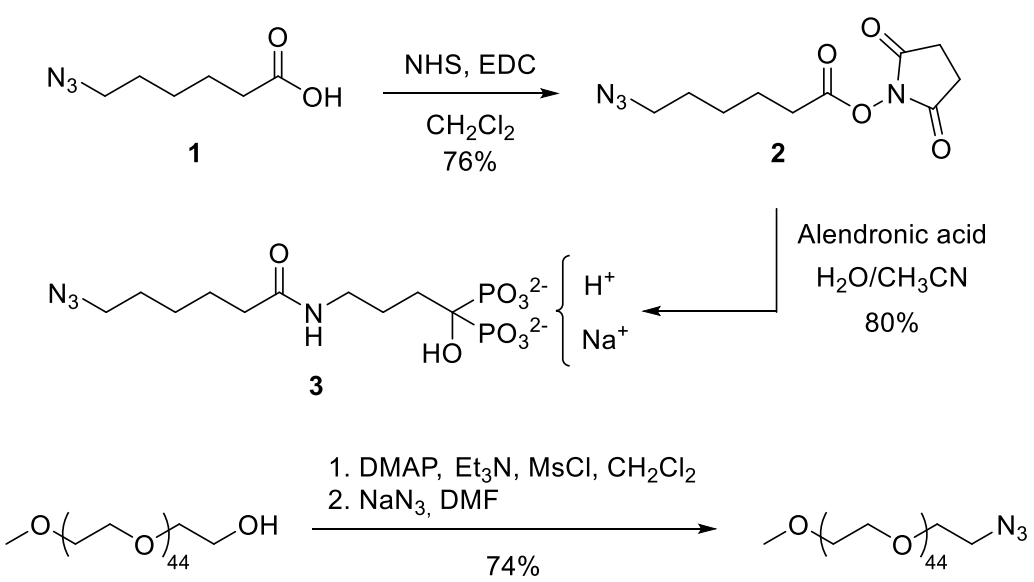

4

Scheme 1. Synthesis of azide derivatives 3 and 4.

First, we synthesized azide alendronate derivative 3 (Scheme 1). The reaction between NHS-ester 2 and alendronic acid monosodium salt was performed in a $\mathrm{MeCN}: \mathrm{H}_{2} \mathrm{O}$ mixture keeping the $\mathrm{pH}$ at $~ 8.5$ in order both to ensure the solubility of the alendronate salt and to guarantee the nucleophilic properties of the primary amine. However, precipitation of the crude after solvent evaporation with EtOH afforded product 3 with $\mathrm{N}$-hydroxysuccinimide (NHS) byproduct, as confirmed by ${ }^{1} \mathrm{H}$ NMR spectra. Attempts to remove NHS by solid-liquid extraction using organic solvents, including EtOAc and $\mathrm{CH}_{3} \mathrm{CN}$, failed. Thus, compound 3 was finally isolated by column chromatography using 5:1 $\mathrm{CH}_{3} \mathrm{CN}: \mathrm{H}_{2} \mathrm{O}$ as initial eluent until NHS was washed out of the column, and $2: 1 \mathrm{CH}_{3} \mathrm{CN}: \mathrm{H}_{2} \mathrm{O}$ for eluting pure 3 in $80 \%$ yield after lyophilization. In the initial alkaline $\mathrm{pH} \sim 8.5$ environment, product 3 should be most likely in a trisodium salt form [26]. However, flash column chromatography probably changed the distribution curves of the species in equilibrium by partial acidification of the mixture. Indeed, negative ESI-TOF mass spectrum depicted the species $[\mathrm{M}-\mathrm{Na}]^{-}$and $[\mathrm{M}-\mathrm{H}]^{-}$ considering $\mathrm{M}$ as the monosodium salt. Similarly, positive ESI-TOF mass spectrum showed $[\mathrm{M}+\mathrm{H}]^{+}$, 
$[\mathrm{M}+\mathrm{Na}]^{+},[\mathrm{M}-\mathrm{H}+2 \mathrm{Na}]^{+}$and $[\mathrm{M}-2 \mathrm{H}+3 \mathrm{Na}]^{+}$, suggesting that the alendronate derivative was obtained mainly as the monosodium salt species.

412 Synthesis of azido PEG chain 4 was performed in two sequential steps. First, commercial 413 MeOPEG ${ }_{45} \mathrm{OH}$, having a methoxy group at one end of the chain, was mesylated and used without 414 any further purification for the reaction with sodium azide in DMF. Azido PEG 4 was isolated by two subsequent filtration processes with THF and $\mathrm{Et}_{2} \mathrm{O}$ that yielded 4 pure enough, as confirmed by NMR analysis. It is important to remark that the broad signal observed at $\delta 3.65-3.61 \mathrm{ppm}$ corresponding to the main chain methylene groups appeared along with ${ }^{13} \mathrm{C}$ satellites due to the ${ }^{13} \mathrm{C}-{ }^{-1} \mathrm{H}$ coupling typically observed in high molecular weight PEG derivatives [27].

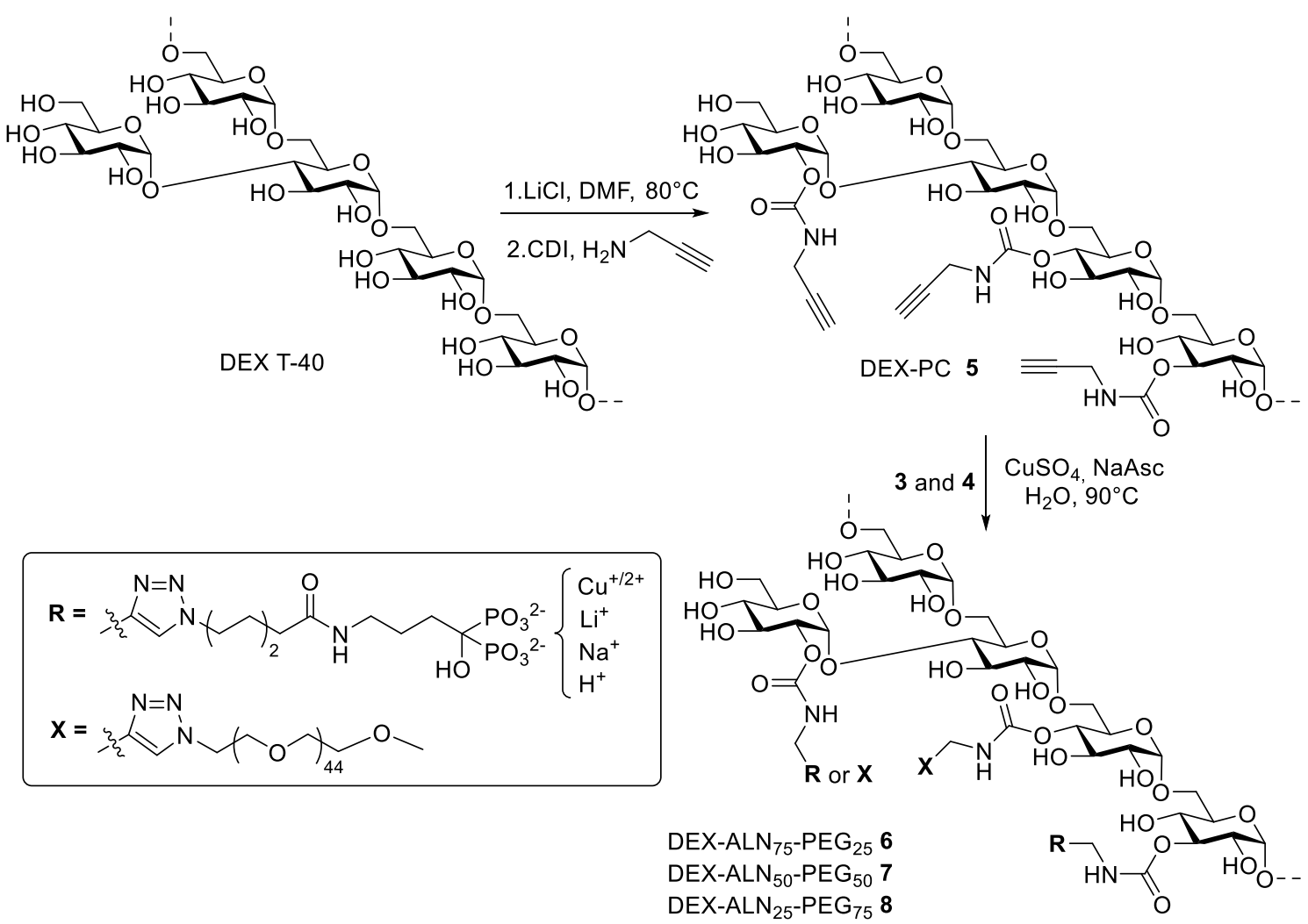

Scheme 2. Synthesis of DEX-PC 5 and DEX-ALN-PEG 6-8. Structures of coplymers 5-8 are intended to simply depict different modes of carbamate attachment to the DEX chain and do not reflect either the actual spatial distribution of the appendages or the degree of substitution along the chain.

The partial alkynylation of the dextran T-40 was achieved upon treatment with carbonyldiimidazole (CDI) and propargylamine forming propargyl carbamate groups on some of the free $\mathrm{OH}$ groups of the polysaccharide [28]. DEX T-40 was extensively dried to minimize unwanted side reactions with water. Reaction was performed in dry DMF in the presence of anhydrous $\mathrm{LiCl}$ to help solubilizing the polysaccharide by interfering with its high crystallinity. DEX T-40 was then subsequently treated with 60 equivalents of CDI and an excess of propargylamine to give DEX-PC 5 (Scheme 2). Taking into account that the number of propargyl carbamate appendages directly depends on the CDI ratio, we expected these amounts to yield a maximum of 60 alkynes per DEX T-40 molecule. After dialysis and lyophilization, the composition of the obtained material was determined by elemental analysis. A mathematical model (see supplementary material) was developed to estimate the number of propargyl groups grafted on the polymer from those data. The model consistently estimated $\sim 50$ propargyl carbamate groups each time the reaction was repeated. The functionalization of the polysaccharide can be observed by ${ }^{1} \mathrm{H}$ NMR through the appearance of broad signals at $\delta 3.03,2.95$ and $2.69 \mathrm{ppm}$ that correspond to the ethynyl proton of propargyl carbamate groups located at 
different positions on the glucose moieties. In addition, a set of new signals arose from the substituted glucose units, namely a doublet at $\delta 5.06 \mathrm{ppm}$ and broad singlet $\delta 4.98$ ppm corresponding to anomeric protons, along with two triplets at $\delta 3.51$ and $3.44 \mathrm{ppm}$ assigned to the H-4 protons. In ${ }^{13} \mathrm{C}$ NMR spectrum, signals at $\delta 157.9-157.0 \mathrm{ppm}$ indicated the presence of carbonyl groups on the structure, while the quaternary and ternary carbons of the ethynyl group gave peaks in the range of $\delta$ 80.4-78.8 and $\delta$ 73.2-73.1 ppm, respectively.

Once DEX-PC 5 was prepared, azides 3 and 4 were conjugated using $\mathrm{CuSO}_{4}$ and sodium ascorbate as catalyst system at $90^{\circ} \mathrm{C}$ in water (Scheme 2 ). Three dextran derivatives with different ratios of ALN and PEG branches were prepared, namely DEX-ALN75-PEG 25 6, DEX-ALN50-PEG50 7 and DEX-ALN25PEG75 8, in order to study the effect of such ratio on both the stability of the coating and the "stealth" abilities of the resulting surface-modified nanoMOFs. To prevent that different diffusion coefficients and reactivity kinetics of structurally so different azides as 3 and 4 might condition the actual ratio on 6-8, conjugations were performed sequentially starting with the required amount of alendronate derivative 3 , followed after 8 hours by addition of an excess of PEG azide 4 . It was expected that the conjugation of the short chain of alendronate in the first place would not hinder the subsequent approach of the much bulkier PEG chain in the second step, which might occur if performed in the opposite order. Purification of 6-8 was carried out by washing with a solution of EDTA at pH $\sim 7$ in order to chelate the excess of copper, followed by dialysis against water using 12-14 KDa MWCO membranes until stable conductivity $(<1 \mu \mathrm{S} / \mathrm{cm})$ was obtained in the dialysate solution. The analysis of number average molecular weights $(M n)$ of polymers before (DEX-PC 5) and after (DEX-ALN50PEG50 7) chemical modification by PEG chains was achieved by SEC-MALLS. The starting material, DEX had a $M n$ value of 31,150 g/mol. It was further shown that grafting ALN and PEG resulted in an expected increase of number average molecular weight with the grafting of PEG chains on the main DEX chain (from 35,240 for DEX-PC 5 to 83,120 g/mol for DEX-ALN50-PEG50 7). This difference of around $50,000 \mathrm{~g} / \mathrm{mol}$ could correspond to a ratio of $25 \%$ of PEG grafted chains of $2000 \mathrm{~g} / \mathrm{mol}$ (since $50 \% \times 2000 \mathrm{~g} / \mathrm{mol} \times 100$ units $=100,000 \mathrm{~g} / \mathrm{mol})$. Nevertheless, note that this determination presents a high uncertainly notably due to the hypothesis that the refractive index increment value of DEX$\mathrm{ALN}_{50}-\mathrm{PEG}_{50}$ was equal to the one of unmodified dextran ( $\left.\mathrm{dn} / \mathrm{dc}=0.147 \mathrm{ml} / \mathrm{g}\right)$. Consequently, elemental analysis and HR-ICP-MS of the obtained copolymers was carried out in order to obtain a more accurate compositional profile for DEX-ALN-PEG derivatives 6-8. The mathematical model constructed to analyze these data (see supplementary material) indicated that only 66-74\% of propargyl carbamates underwent cycloaddition (Table 1). Interestingly, the average number of ALN branches appended to the polysaccharide consistently changed depending on the initial ratio of reagents although in lesser extension than foreseen, varying from $\sim 15$ in the case of DEX-ALN75-PEG 25 6 to $\sim 5$ for DEX-ALN $25-P E G 75$ 8. In sharp contrast, the average number of PEG branches only varies from $\sim 26$ in the case of PEG-ALN75-PEG 256 and $\sim 32$ for DEX-ALN25-PEG75 8. This fact suggests that cycloaddition of PEG azide derivative 4 onto DEX-PC 5 is mainly limited by steric hindrance of the resulting product more than the reagent amount added to the reaction. The robustness of the model is evidenced by the fact that the sum of free propargyl carbamate $(n), \operatorname{ALN}(x)$ and PEG $(z)$ branches keeps around $50( \pm 12 \%)$, which matches the estimated number of propargyl carbamate groups originally present in DEX-PC 5 starting material. It should be underlined that the model predicts the presence of at least one $\mathrm{Cu}$ atom per alendronate branch, along with an extra cation of $\mathrm{Na}$ and/or $\mathrm{Li}$, even after extraction with EDTA prior dialysis. This result is not surprising since it is known that alendronate can chelate $\mathrm{Cu}(\mathrm{II})$ in a wide range of $\mathrm{pH}[29,30]$, and indeed may explain the reduced cycloaddition efficiency for alendronate branches since copper cations could have been sequestered during the reaction. Although free, unbound $\mathrm{Cu}$ (II) has been demonstrated to be toxic for living cells [31], it requires concentrations as high as $78.5 \mu \mathrm{mol} / \mathrm{L}(5 \mathrm{mg} / \mathrm{L})$ in serum for systemic toxicity in humans [32]. Furthermore, the toxicity of chelated copper is remarkably lower, allowing its use to perform CuAAC reactions within living systems [33,34]. We observed that copper present in DEXALN-PEG 6-8 did not hinder MIL-100(Fe) coating (see below) which took place through their alendronate branches, and most likely, it was displaced by iron(III) atoms from nanoMOFs surface 
and washed out during centrifugation isolation. The proposed structures for 6-8 were demonstrated by ${ }^{1} \mathrm{H}$ NMR spectra, where the formation of 1,2,3-triazole residues caused the appearance of a broad signal at $\delta 8.03 \mathrm{ppm}$ in the three cases. In addition, methylene protons of PEG residues gave an intense peak at $\delta 3.73 \mathrm{ppm}$, while weak and broad singlets observed between $\delta 2.25$ and $1.26 \mathrm{ppm}$ evidenced the presence of alendronate branches. Furthermore, signals from non-reacted ethynyl protons were still notorious between $\delta 3.02$ and $2.64 \mathrm{ppm}$, as the compositional model had predicted (Table 1). ${ }^{13} \mathrm{C}$ NMR experiments were strongly dominated by the high signals arising from PEG methylenes, which hindered both their acquisition and assignation. Finally, ${ }^{31} \mathrm{P}$ NMR confirmed the presence of phosphorus nuclei in the molecules with signals at $\delta 0.5-(-2.6) \mathrm{ppm}$.

Table 1. Calculated (see supplementary material) number of free propargyl carbamate $(n)$, alendronate $(x)$ and PEG $(z)$ branches from compositional profiles obtained by elemental analyses and HR-ICP-MS data. ${ }^{a}$

\begin{tabular}{|l|l|l|l|l|}
\hline Derivative & $n$ & $x$ & $z$ & $n+x+z$ \\
& Nb of propargyl groups & Nb of alendronate & Nb of PEG chains & \\
\hline DEX-PC 5 & 50.1 & 0 & -0.1 & 50.1 \\
\hline DEX-ALN75-PEG25 6 & 16.1 & 14.3 & 26.0 & 56.4 \\
\hline DEX-ALN50-PEG50 7 & 13.7 & 10.8 & 30.5 & 55.0 \\
\hline DEX-ALN25-PEG75 8 & 17.6 & 4.9 & 32.3 & 54.8 \\
\hline
\end{tabular}

${ }^{a}$ An error of $\pm 12 \%$ was estimated.

\subsection{MIL-100(Fe) nanoMOFs synthesis and surface modification}

3.2.1 DEX-ALN-PEG coating and physicochemical characterization

MIL-100(Fe) nanoMOFs with a mean hydrodynamic diameter of $191 \pm 23 \mathrm{~nm}$ and BET (Brunauer, Emmett and Teller) surface area of $1690 \pm 80 \mathrm{~m}^{2} \mathrm{~g}^{-1}$ were successfully synthesized by an organic solvent-free microwave-assisted hydrothermal method. They exhibited a facetted morphology (Figure 1C), crystalline structure (Figure 1B) and composition in agreement with previously reported data $[6,12]$.

Surface modification of MIL-100(Fe) nanoMOFs with DEX-ALN-PEG was carried out through a simple, "green" (meaning organic solvent-free) method consisting on simple impregnation of nanoMOFs in aqueous solutions of the synthesized copolymers (Figure 1A). The three copolymers with increased PEG contents, namely DEX-ALN75-PEG25 6, DEX-ALN50-PEG50 7, and DEX-ALN25PEG75 8 were used for the surface modification. 
A
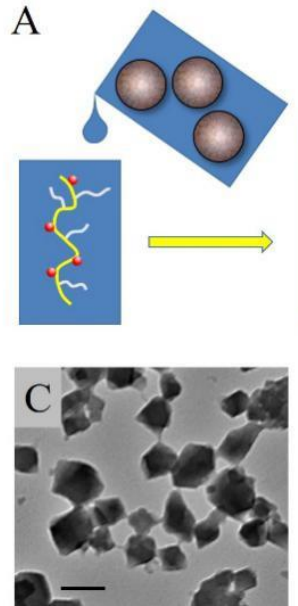

B
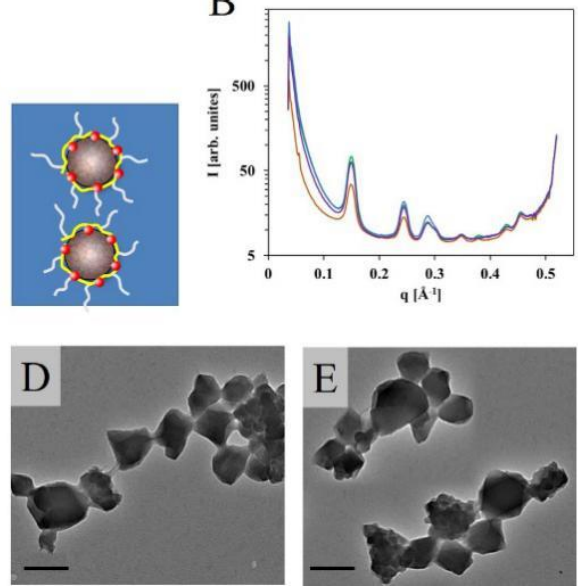

\section{6}

Figure 1. Preparation and characterization of nanoMOFs coated or not with DEX-PEG. A: schematic representation of the «green» preparation of DEX-ALN-PEG coated nanoMOFs in water; B: XRPD patterns of nanoMOFs coated or not with DEX-PEG (orange: nanoMOFs; blue: DEX-ALN50PEG50 coated nanoMOFs; green: DEX-ALN25-PEG75 coated nanoMOFs; purple: DEX-ALN75-PEG25 coated nanoMOFs); C-E: TEM images of uncoated nanoMOFs (C), DEX-ALN50-PEG50 7 coated nanoMOFs (D) and DEX-ALN25-PEG75 8 coated nanoMOFs (E); scale bar (200 nm). The mass ratios DEX-ALN-PEG: nanoMOFs were 1:1.

The amount of DEX-ALN-PEG associated to the nanoMOFs was determined by ICP-MS by direct quantification of the $\mathrm{P}$ content in the coated samples. Indeed, $\mathrm{P}$ is the only element present in the DEX-ALN-PEG copolymers, but not in the nanoMOFs, offering a straightforward and precise quantification method. It was found that after overnight incubation, amounts of DEX-ALN75-PEG 25 6, DEX-ALN50-PEG50 7, and DEX-ALN25-PEG75 8 associated to the nanoMOFs reached $29 \pm 2 \mathrm{wt} \%, 27$ $\pm 2 \mathrm{wt} \%$ and $32 \pm 3 \mathrm{wt} \%$, respectively. Interestingly, these amounts of coating material attached to the nanoMOFs were among the highest reported so far. This efficient association could be possibly attributed to: i) the strong affinity of ALN for iron site at the surface and ii) the cooperative effects of ALN moieties as schematized in Figure 1A. As comparison, cyclodextrin (CD)-phosphate coatings on same MIL-100(Fe) nanoMOFs reached $~ 17 \mathrm{wt} \%$. In that case, only 3-4 phosphate moieties were attached to each CD [35]. Indeed, alendronate, used mainly for osteoporosis treatment, is known to have a strong affinity for metals. It was incorporated in nanoMOFs made of Zr-based UiO-66 NPs [36]. It was highlighted that this amino-bisphosphonate was probably strongly anchored to the $\mathrm{Zr}-\mathrm{O}$ clusters of the UiO NPs, thus promoting both high loading efficiencies (close to $100 \%$ ) and controlled release.

These studies suggest that the amount of DEX-ALN-PEG associated to the nanoMOFs was not significantly affected by the ratio of ALN used in the experiments. This suggest that a large number of ALN moieties are not required for an efficient coating of the nanoMOFs, and that DXT-ALN25PEG75 already shows enough ALN appendages $(\sim 5)$ to perform the most efficient coating we could observed.

The MIL-100(Fe) nanoMOFs coated or not with DEX-ALN-PEG were characterized by a set of complementary techniques. First, XRPD studies indicated that the crystalline structure of MIL100(Fe) nanoMOFs was preserved after surface modification (Figure 1B) in spite of the high amounts of DEX-ALN-PEG associated to the nanoparticles. Second, TEM experiments showed that the nanoMOFs maintained their facetted morphology, regardless of the type of DEX-ALN-PEG adsorbed (Figures 1C-E). 
The hydrodynamic diameters of MIL-100(Fe) nanoMOFs were determined by dynamic light scattering (DLS) in water before and after surface modification. The mean hydrodynamic diameter of nanoMOFs before coating was $191 \pm 23 \mathrm{~nm}$. Whatever the amount and type of DEX-ALN-PEG coating material, there were no significant mean size and polydispersity variations after the coating process. Final mean diameters were in the range of $193 \pm 21 \mathrm{~nm}$ to $209 \pm 31 \mathrm{~nm}$, indicating that the coating thickness was less than $10 \mathrm{~nm}$ and that no aggregation occurred.

Uncoated nanoMOFs had a tendency to aggregate upon storage in water (Figure 2A), in agreement with previous studies [12]. In contrast, nanoMOFs coated with all the DEX-ALN-PEG samples (mass ratio DEX-ALN-PEG:nanoMOFs 1:1) were stable in water up to three weeks storage, showing that their coating efficiently prevented their aggregation. However, the mass ratios DEXALN-PEG:nanoMOFs play an important role on the colloid stability of the nanoMOFs. For example, when reducing 4 fold the amount of DEX-ALN50-PEG50 7 at the nanoMOF surface (mass ratio DEXALN-PEG:nanoMOFs 1:4), aggregation was observed during storage in water, as shown by a diameter increase from $193 \mathrm{~nm}$ to more than $400 \mathrm{~nm}$ in $24 \mathrm{~h}$ (Figure 2A), possibly due to DEX-ALNPEG at the surface of nanoMOFs inducing a bridging effect. Therefore this sample was excluded in the following experiments. All the nanoMOF formulations were stable in the biological medium used in this study (DMEM complemented with 10\% FBS) (Figure 2B). No aggregation was found and the mean diameters remained constant over more than $6 \mathrm{~h}$ incubation at $37^{\circ} \mathrm{C}$, allowing for further biological investigations of interactions with macrophages.
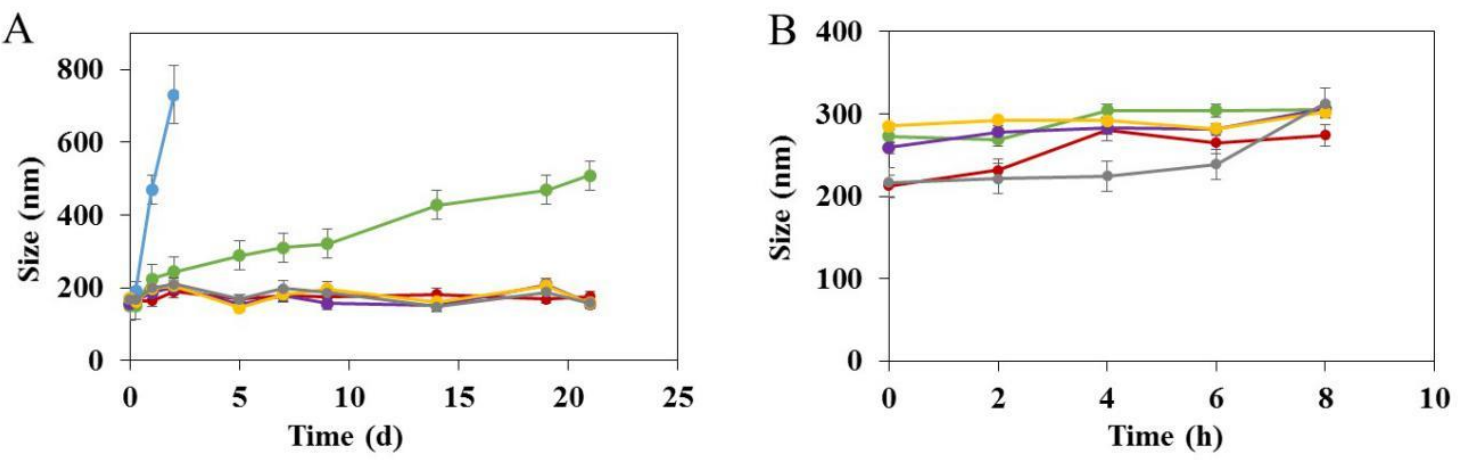

Figure 2. Stability of nanoMOFs coated or not with DEX-ALN-PEG in water (A) and in DMEM cell culture medium (B). Blue: uncoated nanoMOF; purple: nanoMOF coated with DEX-ALN75-PEG25 6 (mass ratio DEX-ALN-PEG:nanoMOFs 1:1); orange: nanoMOF coated with DEX-ALN50-PEG50 7 (mass ratio DEX-ALN-PEG:nanoMOFs 1:1); gray: nanoMOF coated with DEX-ALN25-PEG75 8 (mass ratio DEX-ALN-PEG:nanoMOFs 1:1); green: nanoMOF coated with DEX-ALN50-PEG50 7 (mass ratio DEX-ALN-PEG:nanoMOFs 1:4); red: nanoMOF coated with DEX-ALN50-PEG50 7 (mass ratio DEXALN-PEG: nanoMOFs 1:2).

\subsubsection{Surface properties of nanoMOFs before and after functionalization}

ZP measurements were performed to gain insights on the influence of the coatings on the global charge of the NPs. The ZP of the nanoMOFs, coated or not, was found to be strongly dependent upon the $\mathrm{pH}$ of the suspension medium, in a range of 1 to 10 (Figure 3A). Typically, the ZP of uncoated nanoMOFs was positive $(+23 \pm 3 \mathrm{mV})$ at $\mathrm{pH} 5$, whereas it shifted to negative values $(-15 \pm 3 \mathrm{mV})$ at $\mathrm{pH}$ 7. This was attributed to the presence at the nanoMOFs external surface of both uncoordinated iron sites and terminal $\mathrm{COOH}$ groups from the nanoMOF linker (trimesic acid, $\mathrm{pKa}$ 's $=3.16,3.98$, and 4.85). The ZP values varied after coating with DEX-ALN-PEG copolymers (Figure $3 \mathrm{~A}$ ). At acidic $\mathrm{pH}$ $(<4)$, the $\mathrm{ZP}$ was close to zero $(-3$ to $3 \mathrm{mV})$ indicating that the surface groups on the nanoMOFs were shielded by the coating material. At basic $\mathrm{pH}(>7)$ the $\mathrm{ZP}$ values of $-15 \pm 5 \mathrm{mV}$ were similar to the ones previously reported for DEX coatings [37]. Of note, there was no significant variation for ZP values obtained with the different DEX-ALN-PEG copolymers, except in the case of the copolymer with the 
highest PEG content (8) whose ZP values were the closest to zero. This is in line with the protective effect of PEG shells on NPs, as previously reported [38]. However, when the amount of DEX-ALNPEG copolymers in the nanoMOFs was reduced, the $\mathrm{ZP}$ values at $\mathrm{pH}>7$ tended to approach those of uncoated nanoMOFs (Figure 3A).
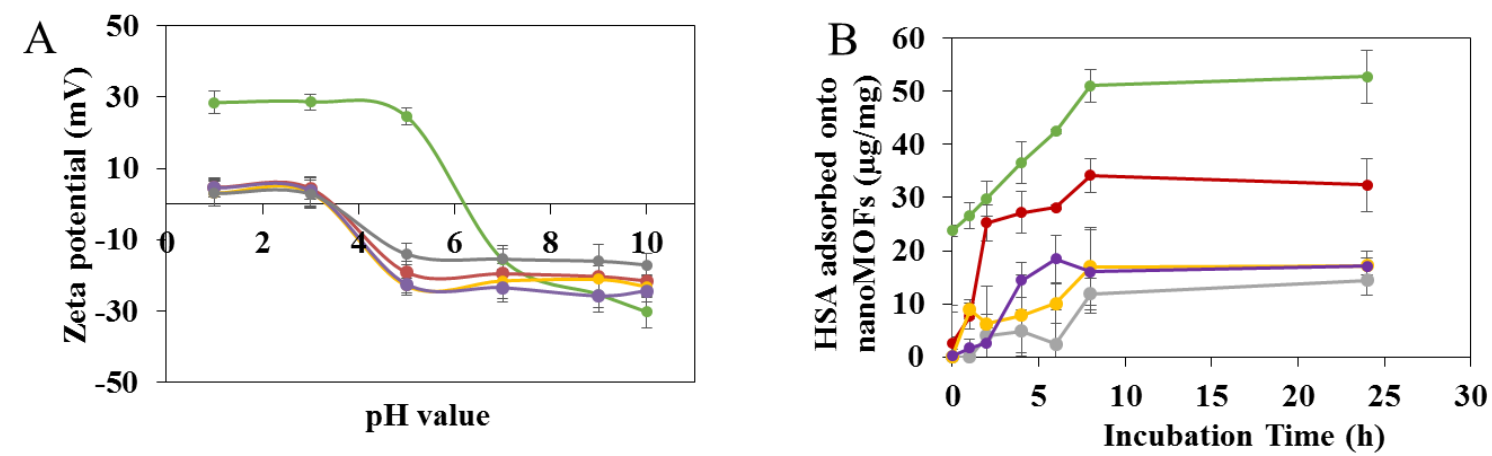

593

Figure 3. Effect of surface modification of nanoMOFs by DEX-ALN-PEG copolymers on their ZP (A) and amounts of HSA adsorbed (B). Green: uncoated nanoMOF; purple: nanoMOF coated with DEX-ALN75-PEG 65 (mass ratio DEX-ALN-PEG: nanoMOFs 1:1); orange: nanoMOF coated with DEX-ALN $50-P E G 507$ (mass ratio DEX-ALN-PEG: nanoMOFs 1:1); gray: nanoMOF coated with DEXALN25-PEG75 8 (mass ratio DEX-ALN-PEG: nanoMOFs 1:1); red: nanoMOF coated with DEX-ALN50PEG50 7 (mass ratio DEX-ALN-PEG: nanoMOFs 1:2).

It is well known that surface functionalization strongly influences the biodistribution of the nanocarriers [10]. Indeed, after intravenous administration of NPs, plasma proteins readily adsorb on the external surface of the NPs, creating the so-called "protein corona", which considerably affects the NPs' physicochemical properties and in vivo fate [39]. To gain further understanding on protein adsorption, the interaction of nanoMOFs coated or not with DEX-ALN-PEG with HSA, the most abundant protein in human blood plasma, was studied here. Indeed, for many other types of NPS, HSA was used as model protein to investigate the capacity of PEG coatings to reduce protein adsorption [39]. NanoMOFs coated or not with DEX-ALN-PEG copolymers were incubated with HSA aqueous solutions and the adsorbed HSA amounts, expressed as $\mu \mathrm{g} / \mathrm{mg}$ of nanoMOFs, were determined (Figure 3B). The experiments were carried out with fixed concentrations of nanoMOFs $(300 \mu \mathrm{g} / \mathrm{mL})$ and HSA $(100 \mu \mathrm{g} / \mathrm{mL})$ aqueous solutions. Non-adsorbed HSA was recovered in the supernatant after centrifugation, followed by quantification using a BCA assay. In the case of uncoated nanoMOFs, the amount of adsorbed HSA reached a plateau within $6 \mathrm{~h}$, with around $50 \mu \mathrm{g}$ HSA/mg nanoMOFs (Figure 3B). Surface modification with DEX-ALN-PEG significantly reduced HSA adsorption, with $\sim 35 \mu \mathrm{g}$ HSA/mg nanoMOFs for the case of DEX-ALN50-PEG50 7 (mass ratio DEX-ALN-PEG:nanoMOFs 1:2) coatings. This amount was further reduced to less than $20 \mu \mathrm{g}$ $\mathrm{HSA} / \mathrm{mg}$ nanoMOFs for the coatings with DEX-ALN75-PEG 25 6, DEX-ALN $50-P E G 50$ 7, and DEX-ALN25PEG75 8 (mass ratio DEX-ALN-PEG:nanoMOFs 1:1). There results clearly show that the DEX-ALNPEG coatings are able to reduce the adsorption of the model protein HSA.

\subsubsection{Macrophage uptake of DEX-ALN-PEG surface modified nanoMOFs}

The capacity of DEX-ALN-PEG coated nanoMOFs to escape macrophage uptake was evaluated on the murine macrophage cell line J774. NanoMOFs were colored using an iron staining procedure (see material and methods), allowing their identification inside cells by optical microscopy. To complete these qualitative studies, quantitative data on the amounts of nanoMOFs internalized in cells were obtained by ICP-MS, after extensive washing to remove the non-associated particles. 
According to an lactic acid dehydrogenase (LDH) test previously used to study nanoMOF toxicity [17], it was shown that the particles used here coated or not with DEX-ALN-PEG shells were nontoxic for the J774 cells up to $100 \mathrm{mg} / \mathrm{mL}$, with more than $80 \%$ cell viability. This is in agreement with previously reported data showing the lack of toxicity of MIL-100(Fe) nanoMOFs $[4,5,14,17,40]$. Internalization kinetics of nanoMOFs in J774 macrophages were studied and a typical example is presented in Figure S14 (supplementary material). The amount of internalized uncoated nanoMOFs was quantified by ICP-MS, showing that after $2 \mathrm{~h}$ incubation $\sim 14 \mu \mathrm{g}$ of uncoated nanoMOFs were taken up in $3 \times 10^{5} \mathrm{~J} 774$ macrophages, corresponding to around $1 \times 10^{7}$ uncoated nanoMOFs/cell and to $47 \%$ of the initial uncoated nanoMOFs (Figure S14). Moreover, this amount almost doubled after $4 \mathrm{~h}$ incubation, reaching $23 \mu \mathrm{g}$ uncoated nanoMOFs $/ 3 \times 10^{5}$ cells $(77 \%$ of the nanoMOFs), thus demonstrating that macrophages avidly take up uncoated nanoMOFs. Remarkably, the presence of the DEX-ALN-PEG coating significantly reduced the nanoMOFs internalization, whatever the incubation time. For example, there was only less than $3 \mu \mathrm{g}$ of coated nanoMOFs in $3 \times 10^{5} \mathrm{~J} 774$ macrophages (corresponding to $10 \%$ of the initial coated nanoMOFs) after $2 \mathrm{~h}$ incubation. Even after $4 \mathrm{~h}$ incubation, the internalized amount of coated nanoMOFs was still less than $25 \%$.

These direct quantification data obtained by ICP MS were supported by optical investigations of the cells after incubation with the nanoMOFs (Figure 4). Before contact with nanoMOFs, J774 presented a homogeneous morphology with round shapes (Figure 4A). A typical image showing the massive uptake of uncoated nanoMOFs is presented in Figure $4 \mathrm{~B}$. After $2 \mathrm{~h}$ incubation, the nanoMOFs appeared clustered inside the macrophages, mostly at their periphery. In contrast, after the same incubation time, the uptake of DEX-ALN75-PEG25 6 coated nanoMOFs was dramatically reduced (Figure 4C).
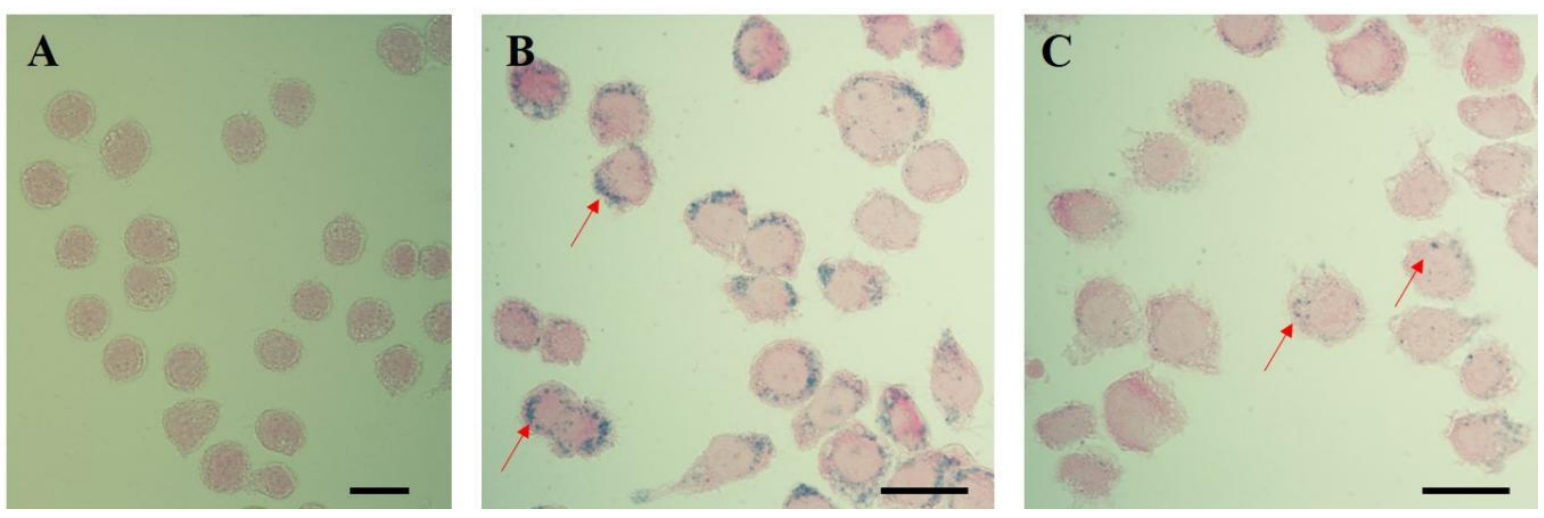

Figure 4. Optical microscopy images showing the effect of DEX-ALN-PEG coatings on J774 macrophage uptake. A: Control J774 macrophages with no treatment; B: J774 macrophages after $2 \mathrm{~h}$ incubation with $50 \mu \mathrm{g} / \mathrm{mL}$ uncoated nanoMOFs; C: J774 macrophages after $2 \mathrm{~h}$ incubation with 50 $\mu \mathrm{g} / \mathrm{mL}$ DEX-ALN75-PEG25 6 coated nanoMOFs (mass ratio DEX-ALN-PEG:nanoMOFs 1:1). Red arrows point out nanoMOFs stained using Prussian blue. Scale bar represents $20 \mu \mathrm{m}$.

The higher uptake of DEX-ALN75-PEG25 6 coated nanoMOFs after $4 \mathrm{~h}$ as compared to $2 \mathrm{~h}$ (Figure S14) could be possibly due to a progressive saturation of the cells and/or a detachment of the coating in the complex biological media containing proteins or by contact with cell membranes. To further compare the different DEX-ALN-PEG coatings, an incubation time of $4 \mathrm{~h}$ was chosen as it corresponds to the blood circulation time of PEG-coated nanoparticles [9]. Figure 5 shows the effect of the DEX-ALN-PEG coatings upon macrophage uptake. The nanoMOFs amounts associated to the cells were 39, 32 and 24\% for DEX-ALN75-PEG 25 6, DEX-ALN50-PEG50 7, and DEX-ALN25-PEG75 8, respectively. This shows that the higher the PEG contents in the coating material, the lower the nanoMOF uptake. 


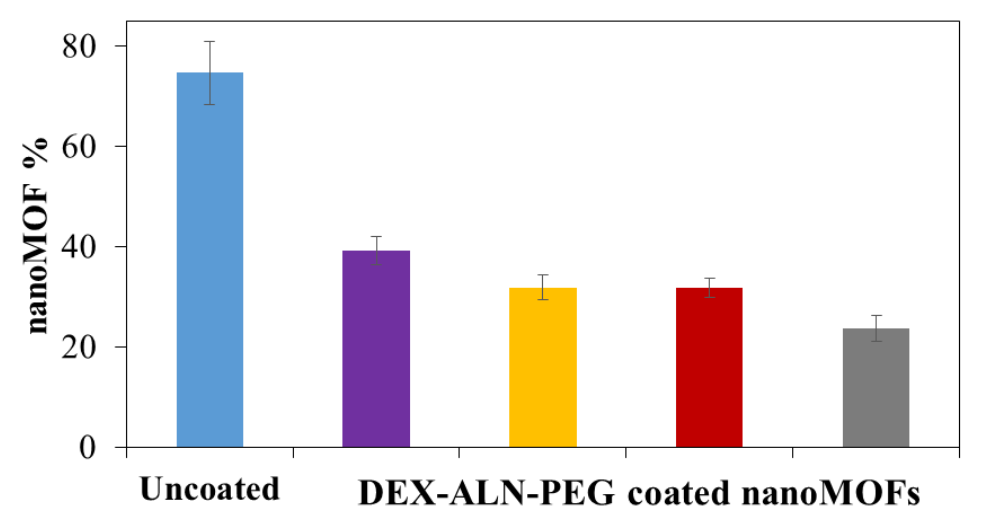

Figure 5. In vitro interaction of nanoMOFs, coated or not with with a J774 macrophage cell line. Purple: nanoMOFs coated with DEX-ALN75-PEG25 6 (mass ratio DEX-ALN-PEG:nanoMOFs 1:1); Orange: nanoMOFs coated with DEX-ALN50-PEG50 7 (mass ratio DEX-ALN-PEG:nanoMOFs 1:1); red: nanoMOFs coated with DEX-ALN50-PEG50 7 (mass ratio DEX-ALN-PEG:nanoMOFs 1:2); gray: nanoMOFs coated with DEX-ALN25-PEG75 8 (mass ratio DEX-ALN-PEG:nanoMOFs 1:1). $50 \mu \mathrm{g} / \mathrm{mL}$ nanoMOFs were incubated with $3 \times 10^{5} \mathrm{~J} 774$ cells for $4 \mathrm{~h}$, then washed to remove the loosely adhering particles. After cell lysis, the amount of internalized nanoMOFs was determined by ICP-MS and was expressed as a \% of the initial amount put in contact with the cells.

To the best of our knowledge, these are the first quantitative data on PEG-coated nanoMOF uptake by macrophages based on a direct quantification and visualization of the Fe content in the cells. In comparison, another recent study dealt with nanoMOF interaction with Raw 246.7 macrophages [14]. In that case, the MIL-100(Fe) nanoMOFs were coated with PEG by GraftFast, whereas a radiolabeled drug, tritiated gemcitabine monophosphate, was incorporated into the particles. In the presence of coated particles, the detected amount of drug in the cells was reduced, as opposed to uncoated ones. However, this study did not take into account drug release during incubation with the cells, which may have influenced the quantity of drug detected as permeated into the cells [19].

\section{Conclusions}

DEX-ALN-PEG copolymers were successfully synthesized, controlling both the PEG and ALN grafting densities ranging from 75:25 to 25:75 ratios. They spontaneously adhered onto the external surface of nanoMOFs in aqueous media, forming a stable coating. In turn, the coating ensured the stability of the nanoMOFs upon storage and enabled to reduce by a 3 fold the in vitro uptake by macrophages. Further studies will deal with the further engineering of the coating by grafting specific ligands and/or fluorescent molecules. The in vivo fate of DEX-ALN-PEG functionalized nanoMOFs will be investigated.

Supplementary Materials: The following are available online at www.mdpi.com/xxx/s1, Figure S1: ${ }^{1} \mathrm{H}$ NMR spectrum $\left(600 \mathrm{MHz}, \mathrm{D}_{2} \mathrm{O}, 25^{\circ} \mathrm{C}\right)$ for compound 3, Figure S2: ${ }^{1} \mathrm{H}$ NMR spectrum $\left(300 \mathrm{MHz}, \mathrm{D}_{2} \mathrm{O}, 25^{\circ} \mathrm{C}\right)$ for compound 4, Figure S3: ${ }^{1} \mathrm{H}$ NMR spectrum $\left(600 \mathrm{MHz}, \mathrm{D}_{2} \mathrm{O}, 25^{\circ} \mathrm{C}\right)$ for DEX-PC 5, Figure S4: ${ }^{1} \mathrm{H}$ NMR spectrum $\left(600 \mathrm{MHz}, \mathrm{D}_{2} \mathrm{O}, 25^{\circ} \mathrm{C}\right)$ for DEX-ALN75-PEG 25 6, Figure S5: ${ }^{1} \mathrm{H}$ NMR spectrum $\left(600 \mathrm{MHz}, \mathrm{D}_{2} \mathrm{O}, 25^{\circ} \mathrm{C}\right)$ for DEXALN50-PEG50 7, Figure S6: ${ }^{1} \mathrm{H}$ NMR spectrum $\left(600 \mathrm{MHz}, \mathrm{D}_{2} \mathrm{O}, 25^{\circ} \mathrm{C}\right)$ for DEX-ALN $25-\mathrm{PEG} 75$ 8, Figure S7: ${ }^{13} \mathrm{C}$ NMR spectrum $\left(150 \mathrm{MHz}, \mathrm{D}_{2} \mathrm{O}, 25^{\circ} \mathrm{C}\right)$ for compound 3, Figure S8: ${ }^{13} \mathrm{C}$ NMR spectrum $\left(75 \mathrm{MHz}, \mathrm{D}_{2} \mathrm{O}, 25{ }^{\circ} \mathrm{C}\right)$ for compound 4, Figure S9: ${ }^{13} \mathrm{C}$ NMR spectrum $\left(150 \mathrm{MHz}, \mathrm{D}_{2} \mathrm{O}, 25^{\circ} \mathrm{C}\right)$ for DEX-PC 5, Figure S10: ${ }^{31} \mathrm{P}$ NMR spectrum (242.9 MHz, $\mathrm{D}_{2} \mathrm{O}, 25^{\circ} \mathrm{C}$ ) for compound 3, Figure S11: ${ }^{31} \mathrm{P}$ NMR spectrum (242.9 MHz, $\left.\mathrm{D}_{2} \mathrm{O}, 25^{\circ} \mathrm{C}\right)$ for DEX-ALN75$\mathrm{PEG}_{25}$ 6, Figure S12: ${ }^{31} \mathrm{P}$ NMR spectrum $\left(242.9 \mathrm{MHz}, \mathrm{D}_{2} \mathrm{O}, 25{ }^{\circ} \mathrm{C}\right)$ for DEX-ALN50-PEG50 7, Figure S13: ${ }^{31} \mathrm{P}$ NMR spectrum $\left(242.9 \mathrm{MHz}, \mathrm{D}_{2} \mathrm{O}, 25^{\circ} \mathrm{C}\right)$ for DEX-ALN25-PEG75 8, Figure S14: Interaction of nanoMOFs, coated or not with DEX-ALN25-PEG75 8, with J774 macrophages. $50 \mu \mathrm{g} / \mathrm{mL}$ nanoMOFs were incubated with $3 \times 10^{5} \mathrm{~J} 774$ cells for $2 \mathrm{~h}$ and $4 \mathrm{~h}$, respectively. 


\section{Author Contributions:}

The author(s) have made the following declarations about their contributions:

Conceived and designed the experiments: RG, AVB, DD

Performed the experiments: GC, JQ, AA, XL, MMM, DF, JMCS, BMA, JRE

Contributed reagents/materials/analysis tools: GC, AA, CL, XL, JQ, MMM, JMCS, DD, RG, AVB

Performed data analysis: GC, RG, AVB, DD, CL, XL, MMM, JMCS

Wrote the paper: RG, AVB, XL, GC

Funding: Financial support for this work was provided by the European Union through FP7-PEOPLE-2013-ITN (http://itn-cyclonhit.eu) project (Grant Agreement no. 608407), the French Agence Nationale de la Recherche (ANR-14-CE08-0017 and ANR-16-CE18-0018), the Spanish Ministry of Economy and Competitiveness (Grant CTQ2017-90050-R) and by government of Principado de Asturias through the project FC-GRUPIN-712 IDI/2018/000166. This work was also supported by a public grant overseen by the French National Research Agency (ANR) as part of the "Investissements d'Avenir" program (Labex NanoSaclay, ANR-10-LABX-0035).

Acknowledgments: We acknowledge Dr. Doru Constantin for help with XRPD experiments. We are grateful to Ludivine Houel Renault for help with the cell culture facility in Centre Laser de l'Université Paris-Sud (CLUPS). This work has benefited from the facilities and expertise of the Liquid Chromatography Platform (Institut de Chimie de Lyon) for the characterization of polymers.

Conflicts of Interest: The authors declare no conflict of interest.

\section{References}

1. Hoskins, B.F.; Robson, R. Infinite polymeric frameworks consisting of three dimensionally linked rod-

2. Horcajada, P.; Gref, R.; Baati, T.; Allan, P.K.; Maurin, G.; Couvreur, P.; Ferey, G; Morris, R. E.; Serre, C. like segments. J. Am. Chem. Soc. 1989, 111, 5962-5964.

3. Yuan, S.; Feng, L.; Wang, K.; Pang, J.; Bosch, M.; Lollar, C.; Sun, Y.; Qin, J.; Yang, X.; Zhang, P. Stable Metal-organic frameworks in biomedicine. Chem. Rev. 2012, 112, 1232-1268.

4. Horcajada, P; Chalati, T.; Serre, C.; Gillet, B.; Sebrie, C.; Baati, T.; Eubank, E.F.; Heurtaux, D.; Clayette, metal-organic frameworks: design, synthesis, and applications. Adv. Mater. 2018, 30, 1-35.

5. Baati, T.; Njim, L.; Neffati, F.; Kerkeni, A.; Bouttemi, M.; Gref, R.; Najjar, M.F.; Zakhama, A.; Couvreur, P.; Kreuz, C.; Chang J. S.; Hwang Y. K.; Marsaud V.; Bories P. N.; Cynober L.; Gil S.; Ferey G.; Couvreur P.; Gref R., Porous metal-organic-framework nanoscale carriers as a potential platform for drug delivery and imaging. Nat. Mater. 2010, 9, 172-178. P.; Serre C. In depth analysis of the in vivo toxicity of nanoparticles of porous iron(III) metal-organic frameworks. Chem. Sci. 2013, 4, 1597-1607.

6. Agostoni, V.; Chalati, T.; Horcajada, P.; Willaime, H.; Anand, R.; Semiramoth, N.; Baati, T.; Hall, S.; Maurin, G.; Chacun, H. An improved anti-HIV activity of NRTI via metal-organic frameworks nanoparticles. Adv. Healthc. Mater. 2013, 2, 1630-1637.

7. Simon-Yarza, T.; Giménez-Marqués, M.; Mrimi, R.; Mielcarek, A.; Gref, R.; Horcajada, P.; Serre, C.; Couvreur, P. A smart metal-organic framework nanomaterial for lung targeting. Angew. Chem. Int. Ed. 2017, 56, 15565-15569. 
8. Simon-Yarza, M.T.; Baati, T.; Paci, A.; Lesueur, L.L.; Seck, A.; Chiper, M.; Gref, R.; Serre, C.; Couvreur, P.; Horcajada, P. Antineoplastic Busulfan encapsulated in metal organic framework nanocarrier: first in vivo results. J. Mater. Chem. B 2016, 4, 585-588.

9. Gref, R.; Minamitake, Y.; Peracchia, M.T.; Trubetskoy, V.; Torchilin, V.; Langer, R. Biodegradable longcirculating polymeric nanospheres. Science 1994, 263, 1600-1603.

10. Gref, R.; Domb, A.; Quellec, P.; Blunk, T.; Müller, R.H.; Verbavatz, J.M.; Langer, R. The controlled intravenous delivery of drugs using PEG-coated sterically stabilized nanospheres. Adv. Drug Deliv. Rev.

11. Barenholz, Y. Doxil ${ }^{\circledR}$ - The first FDA-approved nano-drug: lessons learned. J. Control. Release 2012, 160, 117-134.

12. Agostoni, V.; Horcajada, P.; Noiray, M.; Malanga, M.; Aykaç, A.; Jicsinszky, L.; Vargas-Berenguel, A.; Semiramoth, N.; Daoud-Mahammed, S.; Nicolas, V.; et al. A "green" strategy to construct non-covalent, stable and bioactive coatings on porous MOF nanoparticles. Sci. Rep. 2015, 5, 7925.

13. Zhang, H.H.; Hu, H.; Zhang, H.H.; Dai, W.; Wang, X.; Wang, X.; Zhang, Q. Effects of PEGylated paclitaxel nanocrystals on breast cancer and its lung metastasis. Nanoscale 2015, 7, 10790-10800.

14. Giménez-Marqués, M.; Bellido, E.; Berthelot, T.; Simón-Yarza, T.; Hidalgo, T.; Simón-Vázquez, R.; González-Fernández, Á.; Avila, J.; Asensio, M.C.; Gref, R.; et al. GraftFast surface engineering to improve MOF nanoparticles furtiveness. Small 2018, 14, 1801900.

15. Chen, D.; Yang, D.; Dougherty, C.A.; Lu, W.; Wu, H.; He, X.; Cai, T.; Van Dort, M.E.; Ross, B.D.; Hong, H. In vivo targeting and positron emission tomography imaging of tumor with intrinsically radioactive metal-organic frameworks nanomaterials. ACS Nano 2017, 11, 4315-4327.

16. Zimpel, A.; Preiß, T.; Röder, R.; Engelke, H.; Ingrisch, M.; Peller, M.; Rädler J.O.; Wagner, E.; Bein T.; Lächelt, U.; et al. Imparting functionality to MOF nanoparticles by external surface selective covalent attachment of polymers. Chem. Mater. 2016, 28, 3318-3326.

17. Li, X.; Semiramoth, N.; Hall, S.; Tafani, V.; Josse, J.; Laurent, F.; Salzano, G.; Foulkes, D.; Brodin, P.; Majlessi, L.; et al. Compartmentalized encapsulation of two antibiotics in porous nanoparticles: an efficient strategy to treat intracellular infections. Part. Part. Syst. Charact. 2019, 36, 1800360.

18. Di Nunzio, M.R.; Agostoni, V.; Cohen, B.; Gref, R.; Douhal, A. A “ship in a bottle" strategy to load a hydrophilic anticancer drug in porous metal organic framework nanoparticles: Efficient encapsulation,

19. Rodriguez-Ruiz, V.; Maksimenko, A.; Anand, R.; Monti, S.; Agostoni, V.; Couvreur, P.; Lampropoulou, in metal-organic framework nanoparticles. J. Drug Target. 2015, 23, 759-767. 

organic framework MIL-100(Fe) nanoparticles. J. Mater. Chem. B 2013, 1, 4231-4242.

21. Simon-Yarza, T.; Baati, T.; Neffati, F.; Njim, L.; Couvreur, P.; Serre, C.; Gref, R.; Najjar, M.F.; Zakhama, A.; Horcajada, P. In vivo behavior of MIL-100 nanoparticles at early times after intravenous administration. Int. J. Pharm. 2016, 511, 1042-1047.

22. Perrin, D.D.; Armarego, W.F.L. Purification of Laboratory Chemicals, 3rd ed., Pergamon: Oxford, 1989.

23. Alemán, E.A.; Pedini, H.S.; Rueda, D. Covalent bond-based immobilization approaches for single-

24. Kuil, J.; Branderhorst, H.M.; Pieters, R.J.; De Mol, N.J.; Liskamp, R.M.J. ITAM-derived phosphopeptidecontaining dendrimers as multivalent ligands for Syk tandem SH2 domain. Org. Biomol. Chem. 2009, 7,

25. Grandjean, C.; Boutonnier, A.; Guerreiro, C.; Fournier, J.M.; Mulard, L.A. On the preparation of carbohydrate-protein conjugates using the traceless Staudinger ligation. J. Org. Chem. 2005, 70, 71237132.

26. Ke, J.; Dou, H.; Zhang, X.; Uhagaze, D.S.; Ding, X.; Dong, Y. Determination of pKa values of alendronate sodium in aqueous solution by piecewise linear regression based on acid-base potentiometric titration. J. Pharm. Anal. 2016, 6, 404-409.

27. Semple, J.E; Sullivan, B.; Vojkovsky, T.; Sill, K.N. Synthesis and facile end-group quantification of functionalized PEG azides. J. Polym. Sci. Part A Polym. Chem. 2016, 54, 2888-2895.

28. Sun, G.; Lin, X.; Wang, Z.; Feng, Y.; Xu, D.; Shen, L. PEGylated inulin as long-circulating pharmaceutical

29. Ostović, D.; Stelmach, C.; Hulshizer, B. Formation of a chromophoric complex between alendronate and copper(II) ions. Pharm. Res. 1993, 10, 470-472.

30. Oms, O.; Yang, S.; Salomon, W.; Marrot, J.; Dolbecq, A.; Rivière, E.; Bonnefont, A.; Ruhlmann, L.; Mialane, P. Heteroanionic materials based on copper clusters, bisphosphonates, and polyoxometalates: magnetic properties and comparative electrocatalytic $\mathrm{NO}_{x}$ reduction studies. Inorg. Chem. 2016, 55, 15511561.

801 31. Biaglow, J.E.; Manevich, Y.; Uckun, F.; Held, K.D. Quantitation of hydroxyl radicals produced by radiation and copper- linked oxidation of ascorbate by 2-deoxy-D-ribose method. Free Radic. Biol. Med. 1997, 22, 1129-1138.

$804 \quad$ 32. Barceloux, D.G. Copper. Clin. Toxicol. 1999, 37, 217-230. 
34. Su, Y.; Li, L.; Wang, H.; Wang, X.; Zhang, Z. All-in-One azides: empowered click reaction for in vivo labeling and imaging of biomolecules. Chem. Commun. 2016, 52, 2185-2188.

35. Aykaç, A.; Noiray, M.; Malanga, M.; Agostoni, V.; Casas-Solvas, J.M.; Fenyvesi, É.; Gref, R.; VargasBerenguel, A. A non-covalent "click chemistry" strategy to efficiently coat highly porous MOF nanoparticles with a stable polymeric shell. Biochim. Biophys. Acta - Gen. Subj. 2017, 1861, 1606-1616. efficient capture of alendronate and its mediated release. Chem. Commun. 2014, 50, 8779-8782.

814 37. Lemarchand, C.; Gref, R.; Lesieur, S.; Hommel, H.; Vacher, B.; Besheer, A.; Maeder, K.; Couvreur, P. Physico-chemical characterization of polysaccharide-coated nanoparticles. J. Control. Release 2005, 108, 97-111.

817 38. Rouzes, C.; Gref, R.; Leonard, M.; De Sousa Delgado, A.; Dellacherie, E. Surface modification of poly(lactic acid) nanospheres using hydrophobically modified dextrans as stabilizers in an o/w emulsion/evaporation technique. J. Biomed. Mater. Res. 2000, 50, 557-565.

820 39. Gref, R.; Lück, M.; Quellec, P.; Marchand, M.; Dellacherie, E.; Harnisch, S.; Blunk, T.; Müller, R.H. "Stealth" corona-core nanoparticles surface modified by polyethylene glycol (PEG): Influences of the corona (PEG chain length and surface density) and of the core composition on phagocytic uptake and plasma protein adsorption. Colloids Surf. B Biointerfaces 2000, 18, 301-313. González-Fernández, Á.; Serre, C.; Alonso, M.J.; Horcajada, P. Heparin-engineered mesoporous iron 